\title{
A PROBABILISTIC HARNACK INEQUALITY AND STRICT POSITIVITY OF STOCHASTIC PARTIAL DIFFERENTIAL EQUATIONS
}

\begin{abstract}
ZHENAN WANG
ABSTRACT. Under general conditions we show an a priori probabilistic Harnack inequality for the non-negative solution of a stochastic partial differential equation of the following form

$$
\partial_{t} u=\operatorname{div}(\mathbb{A} \nabla u)+f(t, x, u ; \omega)+g_{i}(t, x, u ; \omega) \dot{w}_{t}^{i} .
$$

We will also show that the solution of the above equation will be almost surely strictly positive if the initial condition is non-negative and not identically vanishing.
\end{abstract}

\section{INTRODUCTION}

Stochastic partial differential equations (SPDEs) have been studied extensively during the last four decades. Fine properties for the solutions have always been a difficult topic. On the topic of positivity for the solution of linear SPDEs with multiplicative noise, it is well known since the beginning that the solution will remain non-negative if the initial condition is non-negative, see Krylov [6] and Pardoux [10]. As for the strictly positivity of the solution, the question for stochastic heat equation is addressed by Carl Mueller [8] in 1991. In their work in 1998, [11], Tessitore and Zabczyk have extended the result to a form that is more general. The strict positivity question can also be asked for non-linear SPDEs such as the ones studied in Debussche, De Moor and Hofmanova [2] and Pardoux [9]. In particular, many examples of semi-linear SPDEs with measurable coefficients can be found in the survey monograph edited by Carmona and Rozovskii [1] and the answer to the strict positivity question for these equations is also unknown. The goal of this paper is to address such problem for a class of semi-linear SPDEs.

In the paper we consider the following type of SPDEs on $\mathbb{R}^{n}$ :

$$
\partial_{t} u=\operatorname{div}(\mathbb{A} \nabla u)+f(t, x, u ; \omega)+g_{i}(t, x, u ; \omega) \dot{w}_{t}^{i}
$$

where $\left\{w^{i}\right\}$ is a sequence of independent standard Brownian motions on a filtered probability space $\left(\Omega, \mathscr{F}_{*}, \mathbb{P}\right)$ and $g=\left\{g_{i}\right\}$ is an $\ell^{2}$-valued function such that for each fixed $x$ and an $\mathscr{F}_{*}=\left\{\mathscr{F}_{t}\right\}$-progressively measurable process $h$, the process $g\left(t, x, h_{t} ; \omega\right)$ is also progressively measurable. We will show a probabilistic Harnack inequality for non-negative solutions of such equation and use the inequality to conclude that the solution stays strictly positive if the initial condition is non-negative and not identically vanishing. The probabilistic Harnack inequality is a local result, therefore we work on a domain $B$ in $\mathbb{R}^{n}$ along a time interval $I$ starting at 0 . The basic assumptions are as follows:

(1) uniform ellipticity: $\mathbb{A}(t, x, u ; \omega)$ is $\mathscr{F}_{*}$-progressively measurable and uniformly elliptic on the space-time domain on which the solution lies, i.e., there is a positive constant $\iota$ such that

$$
\iota \mathrm{Id} \leq \mathbb{A}(t, x, u ; \omega) \leq \iota^{-1} \mathrm{Id}, \quad \forall(t, x, u, \omega) \in I \times B \times \mathbb{R} \times \Omega .
$$

(2) linear growth near $\infty$ and linear decay near 0: there exists a positive constant $\Lambda$ such that

$$
|f(t, x, u ; \omega)|+|g(t, x, u ; \omega)|_{\ell^{2}} \leq \Lambda|u|, \quad \forall(t, x, u ; \omega) \in I \times B \times \mathbb{R} \times \Omega .
$$

We emphasize that no further conditions concerning the continuity $A, f$ or $g$ are imposed. 
A function $u=u(t, x ; \omega)$ is said to be a (stochastically strong) solution of (1.1) on $I \times B$ if $u$ is almost surely a $L^{\infty}\left(I, L^{2}(B)\right)$ process, lives in $L^{2}\left(\Omega \times I, W^{1,2}(B)\right)$ and satisfies the corresponding partial differential equation (PDE) in the sense that

$$
\langle u(t), \varphi\rangle=\langle u(0), \varphi\rangle-\int_{0}^{t}\langle\mathbb{A} \nabla u(s), \nabla \varphi\rangle d s+\int_{0}^{t}\langle f(u(s)), \varphi\rangle d s+\int_{0}^{t}\left\langle g_{i}(u(s)), \varphi\right\rangle d w_{s}^{i}
$$

for all $\varphi \in C_{c}^{\infty}(B)$. Here $\langle\cdot, \cdot\rangle$ denotes the standard inner product on $L^{2}\left(\mathbb{R}^{n}\right)$. The probabilistic Harnack inequality is described in the following theorem.

Theorem 1.1 (Probabilistic Harnack inequality). Let $U=I \times B$ be a bounded space-time rectangle and $u$ be a non-negative solution of (1.1) on $U$. Let $P$ and $Q$ be two bounded space time domains as shown in Figure 1, namely, $P$ is strictly after $Q$ in time, $Q$ is strictly after 0 and both are contained in $U$. Then for any $\epsilon>0$, we have a constant $\Gamma_{0}$ depending only on $n, l, \Lambda$ and the positions of $P$ and $Q$, such that for all $\Gamma>\Gamma_{0}$ and $a>0$,

$$
\mathbb{P}\left\{\sup _{Q} u>a, \quad \Gamma \inf _{P} u \leq a\right\} \leq \epsilon
$$

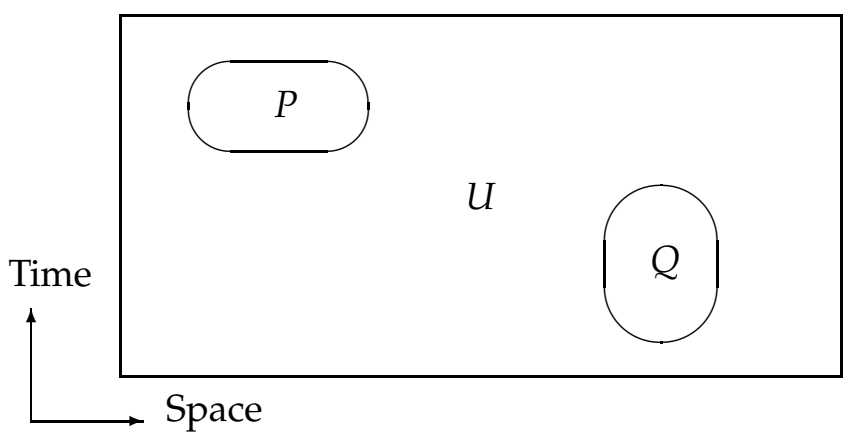

FIGURE 1. Relative positions of $P, Q$ and $U$.

Using this probabilistic Harnack inequality, we can show the strict positivity for (1.1).

Theorem 1.2. Let $u$ be a solution of the SPDE (1.1) on $\mathbb{R}^{+} \times \mathbb{R}^{n}$ with a (deterministic) non-negative and not identically vanishing initial condition $u(0)=u_{0} \in L^{2}\left(\mathbb{R}^{n}\right)$. Then almost surely, $u(t, x)>0$ for all $x \in \mathbb{R}^{n}$ and $t>0$.

The methods we use in this article are drastically different from the conventional approaches used for positivity problems of SPDEs. We continue our work in [5] and combine ideas from Fabes and Garofalo [3] and Moser [7]. Rather than relying on the solution kernel, we analyze the local behavior of the energy for the solution by a combination of PDE techniques and stochastic analysis. Our work can be viewed as a stochastic version of Moser's work including a stochastic version of the time-lagged bounded mean oscillation property, therefore our flexible method can potentially be further applied to other type of nonlinear SPDEs.

The paper is organized as follows. In SECTION 2, we will present a four-step outline of the proof for THEOREM 1.1, complete the proofs for the first and fourth steps in the outline, and prove THEOREM 1.2. In SECTIONS 3, 4 and 5, we will give proofs for the second step. In SECTIONS 6, we will give the proofs for the third step. 


\section{OUTLINES OF THE PROOF}

In this section, we will first outline the proof for the deterministic parabolic Harnack inequality, and then develop a parallel process for THEOREM 1.1. In the following, we use $\|\cdot\|_{p, D}$ to denote the $L^{p}$ norm on a domain $D$ in $\mathbb{R}^{n}$ or $\mathbb{R}^{+} \times \mathbb{R}^{n}$; thus $\|f\|_{p, D}=\left(\int_{D} f^{p} d x\right)^{\frac{1}{p}}$ or $\|f\|_{p, D}=\left(\int_{D} f^{p} d x d t\right)^{\frac{1}{p}}$, depending on the context.

With the same picture as in FIGURE 1, Moser [7] established the deterministic Harnack inequality for parabolic equations as follows.

Theorem 2.1 (Moser's parabolic Harnack inequality). Let $u$ be a non-negative solution of the parabolic equation

$$
\frac{\partial u}{\partial t}=\sum_{k, l=1}^{n} \frac{\partial}{\partial x_{k}}\left(a_{k l}(t, x) \frac{\partial u}{\partial x_{l}}\right)
$$

in $U$ with $\left(a_{k l}\right)$ uniformly elliptic. For $P$ and $Q$ satisfying the same requirement as in THEOREM 1.1 there exists a constant $C$ depending only on $\left(a_{k l}\right)$ such that

$$
\sup _{Q} u \leq \inf _{P} u
$$

Moser's method establishes the inequality in the following four steps, as shown in FIGURE 2

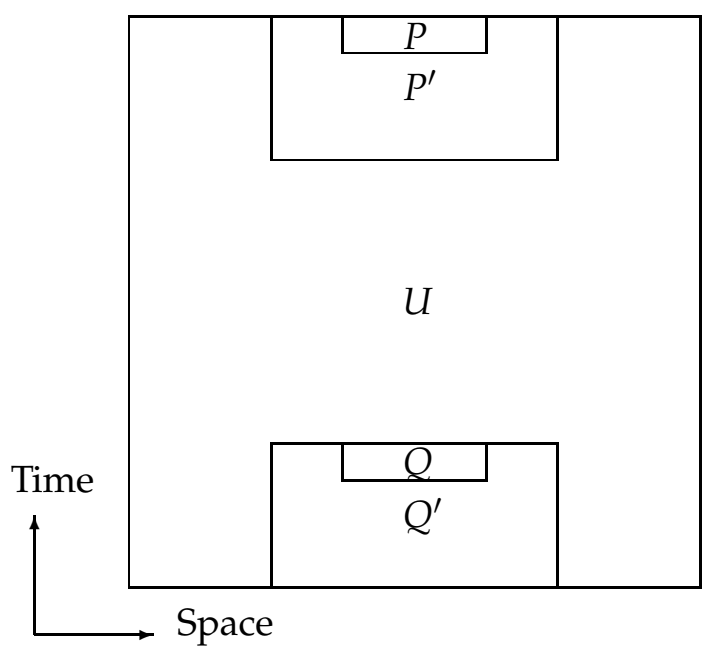

FIGURE 2. Relative positions of $P, Q, P^{\prime}, Q^{\prime}$ and $U$.

(1) We choose $P^{\prime}$ and $Q^{\prime}$ to be slightly larger than of $P$ and $Q$ respectively, $P^{\prime}$ need to be strictly after $Q^{\prime}$ while $Q^{\prime}$ is allowed to touch time 0 , the sizes of the rectangles in FIGURE 2 are exaggerated.

(2) For a fixed $\mu>0,1 /(u+\mu)$ is a sub-solution of (2.1). The De Giorgi iteration scheme shows that for all $p>0$, there exists $C_{p}>0$ such that $\sup _{P}\{1 /(u+\mu)\} \leq C_{p}\|1 /(u+\mu)\|_{p, P^{\prime}}$; at the same time $u+\mu$ is a solution of (2.1), the same process gives $\sup _{Q}\{u+\mu\} \leq$ $C_{p}\|u+\mu\|_{p, Q^{\prime}}$.

(3) Now $-\log (u+\mu)$ is a sub-solution of an equation of the same type as (2.1). This fact guarantees bounded mean oscillation (BMO) property in the parabolic sense for $\log (u+\mu)$. 
The parabolic John-Nirenberg inequality gives $\|1 /(u+\mu)\|_{p_{0}, P^{\prime}}\|u+\mu\|_{p_{0}, Q^{\prime}} \leq K$ for some $p_{0}$ and $K$ independent of $\mu$.

(4) Combining the results in the above two steps with $p=p_{0}$ in the second step, we have $\inf _{P} u \geq C_{p_{0}}^{-2} K^{-1} \sup _{Q} u$ after letting $\mu \rightarrow 0$.

To properly develop a stochastic version of Moser's method, we have to make two major difficulties. The first one is the lack of a definition of stochastic sub-solutions. It turns out that the naïve thought of simply changing the equality in the definition of the solutions to ' $\leq$ ' is insufficient, as we need to describe the martingale property of the sub-solutions. We define a sub-solution as follows.

Definition 2.2. An almost surely bounded $L^{2}(B)$ process $u$ on I living in $L^{2}\left(\Omega \times I, W^{1,2}(B)\right)$ is a (stochastically strong) sub-solution of (1.1) on $I \times B$ if for all non-negative function $\phi \in C_{c}^{\infty}(B)$ and $s \leq t$,

(1) $\langle u(t)-u(s), \phi\rangle \leq-\int_{s}^{t}\langle\mathbb{A} \nabla u(\tau), \nabla \phi\rangle d \tau+\int_{s}^{t}\langle f(\tau), \phi\rangle d \tau+\int_{s}^{t}\left\langle g_{i}(\tau), \phi\right\rangle d w_{\tau}^{i} ;$

(2) the quadratic variation process of $\langle u, \phi\rangle$ at time $t$ equals to $\sum_{i} \int_{0}^{t}\left\langle g_{i}^{2}(\tau), \phi^{2}\right\rangle d \tau$.

Here, $\langle\cdot, \cdot\rangle$ denotes the standard inner product on $L^{2}(B)$.

The next difficulty is establishing stochastic version of inequalities in Moser's proof. Using a random variable $X$, say a certain norm of the solution $u$ of (1.1), to bound another random variable $Y$, say another norm of $u$, with a non-random coefficient usually turns out impractical in the stochastic setting. Indeed, in our case, we cannot expect such kind of estimate to hold for norms of $u$ path-wise. Instead, to resemble the deterministic inequality $X \leq C Y$, we use the tail probability of $Y$ to control the tail probability of $X$, namely,

$$
\mathbb{P}\{X>a, C Y \leq a\}=o(1) \text { as } C \rightarrow \infty \text { for all } a>0 .
$$

At this point, we need to fix a few notations. For technical reasons, we use the maximum norm on $\mathbb{R}^{n}$, i.e., $|x|:=\max _{i}\left\{\left|x_{i}\right|\right\}$. We use the notation $B_{r}\left(x_{0}\right):=\left\{x \in \mathbb{R}^{n}|| x-x_{0} \mid<r\right\}$. A $B_{r}$ without specifying the center will be understood as $B_{r}(0)$. We also define $Q_{r}\left(t_{0}, x_{0}\right)$ as the space-time rectangular region $\left(t_{0}-r^{2}, t_{0}\right] \times B_{r}\left(x_{0}\right)$. A $Q_{r}$ without specifying the base point will be understood as $Q_{r}(1,0)$.

For a rectangular region $I \times B$, we define the following norms for all $p$ and $q$ positive,

$$
\|h\|_{p, q, I \times B}:=\|h\|_{L^{p}\left(I, L^{q}(B)\right)}=\left(\int_{I}\|h\|_{q, B}^{p} d t\right)^{1 / p} .
$$

In SEction 3, we will develop a local version of stochastic De Giorgi iteration from [5] and prove the following result.

Proposition 2.3. Let $u$ be a sub-solution of (1.1) in $Q_{1}$. Then there exist $\Gamma(0)$ and $\delta(0)$ depending only on $n, \iota$ and $\Lambda$ such that for all $a>0, r \in(0,1]$, and $\Gamma \geq \Gamma(0)$,

$$
\mathbb{P}\left\{\|u\|_{\infty, Q_{r / 2}}>a,(r / 2)^{-(n+1) / 2}\|u\|_{4,2, Q_{r}} \leq a / \Gamma\right\} \leq \exp \left\{-\Gamma^{\delta(0)} / r^{2}\right\} .
$$

The positions of $Q_{r / 2}$ and $Q_{r}$ are shown in FIGURE 3

In SECTIONS 4 and 5, we will strengthen the above result into the following form, which will be used to prove the stochastic analogy of the second step in Moser's method.

Proposition 2.4. Let $u$ be a sub-solution to (1.1) in $Q_{1}$. For every $2 \geq p>0$, there exist $\Gamma(p), \delta(p)$ depending only on $n, l, \Lambda$ and $p$ such that for all $a>0, \Gamma \geq \Gamma(p)$ and $0<r<R \leq 1$,

$$
\mathbb{P}\left\{\|u\|_{\infty, Q_{r}}>a,(R-r)^{-(n+2) / p}\|u\|_{p, Q_{R}} \leq a / \Gamma\right\} \leq \exp \left\{-\Gamma^{\delta(p)} / R^{2}\right\} .
$$


$(1,0)$

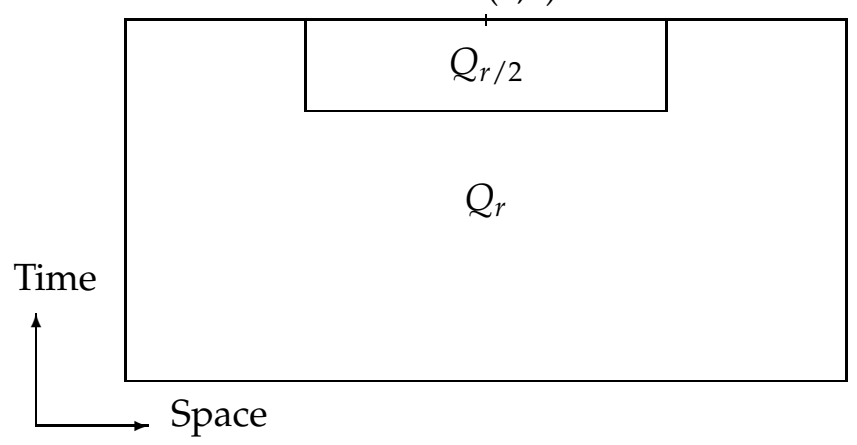

FIGURE 3. Relative positions of $Q_{r / 2}$ and $Q_{r}$.

To make our presentation for the analogy of the third step clearer, we will use the following notation from now on for any function $v>0$ and bounded measurable regions $D_{1}$ and $D_{2}$,

$$
\mathcal{F}[v, \alpha]_{D_{1}, D_{2}}:=\left(\int_{D_{1}} v^{-\alpha} d x d t\right)\left(\int_{D_{2}} v^{\alpha} d x d t\right) .
$$

In SECTIONS 6, we will provide a variant of the parabolic John-Nirenberg inequality in [3, Theorem 1]. We then use this variant to prove the following reverse Cauchy-Schwarz inequality type statement.

Proposition 2.5. Given $t \in(0,1)$, for every $\epsilon>0$, there exist constants $\alpha_{\epsilon}$ and $K_{\epsilon}$ depending only on $n, l, \Lambda, t$ and $\epsilon$ such that $\forall \mu>0$ and any non-negative super-solution $u$ of $(1.1)$ in $[0,2] \times B_{1}$.

$$
\mathbb{P}\left\{\mathcal{F}\left[u+\mu, \alpha_{\epsilon}\right]_{D^{+}, D^{-}}^{1 / \alpha_{\epsilon}}>K_{\epsilon}\right\}<\epsilon .
$$

Here, $D^{+}=\left(2-t^{2}, 2\right) \times B_{t}$ and $D^{-}=\left(0, t^{2}\right) \times B_{t}$ as shown in FIGURE 4

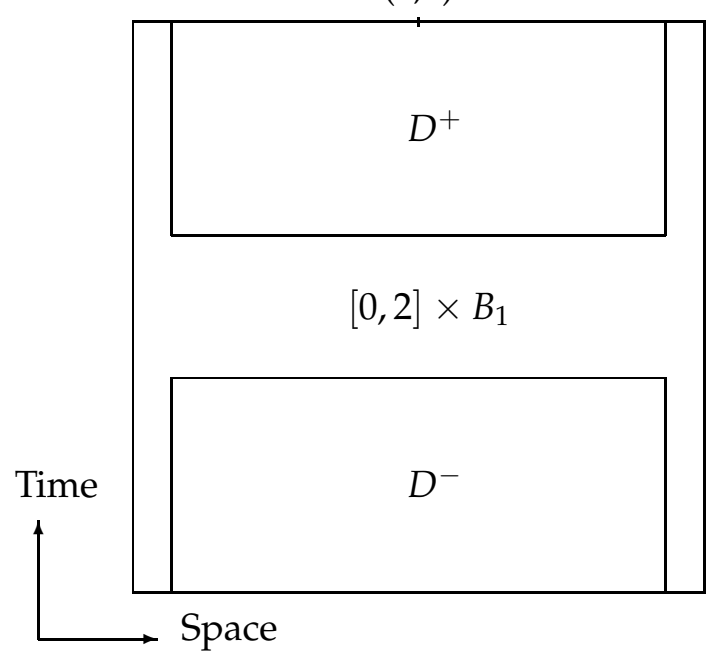

FIGURE 4. Relative positions of $D^{+}, D^{-}$and $[0,2] \times B_{1}$.

Now we prove the probabilistic Harnack inequality. For ease of reference, we restate it here. 
Theorem 2.6. Let $U=I \times B$ be a bounded space-time rectangle and let $P$ and $Q$ be two bounded space time domains as shown in Figure 1, namely, $P$ is strictly after $Q$ in time, $Q$ is strictly after 0 and both are contained in $U$. Then for any $\epsilon>0$, we have a constant $\Gamma_{0}$ depending only on $n, l, \Lambda$ and the positions of $P$ and $Q$, such that for all $\Gamma>\Gamma_{0}, a>0$ and any non-negative solution $u$ of (1.1) on $U$.

$$
\mathbb{P}\left\{\sup _{Q} u>a, \quad \Gamma \inf _{P} u \leq a\right\} \leq \epsilon .
$$

Proof. Without loss of generality, we first enlarge $P$ and $Q$ to be two space-time rectangular regions of the form $I_{P} \times B^{\prime}$ and $I_{Q} \times B^{\prime}$. We still require $I_{P}$ strictly after $I_{Q}$ and $I_{Q}$ strictly after 0 .

We will now consider two separate cases and prove the theorem for each of them.

Case I. $I_{P}$ and $I_{Q}$ have the same length.

With proper scaling and translation, we can now assume $U$ contains $[0,2] \times B_{1}$ and pick up an $r \in(0,1)$ such that $P$ is contained in $Q_{r}(2,0)$ and $Q$ is contained in $Q_{r}(r, 0)$.

We choose $R=\sqrt{r}>r$, then we have the inclusions $P \subset Q_{r}(2,0) \subset Q_{R}(2,0) \subset Q_{1}(2,0)$ and $Q \subset Q_{r}(r, 0) \subset Q_{R}(r, 0) \subset Q_{1}$, as shown in FIGURE 5 .

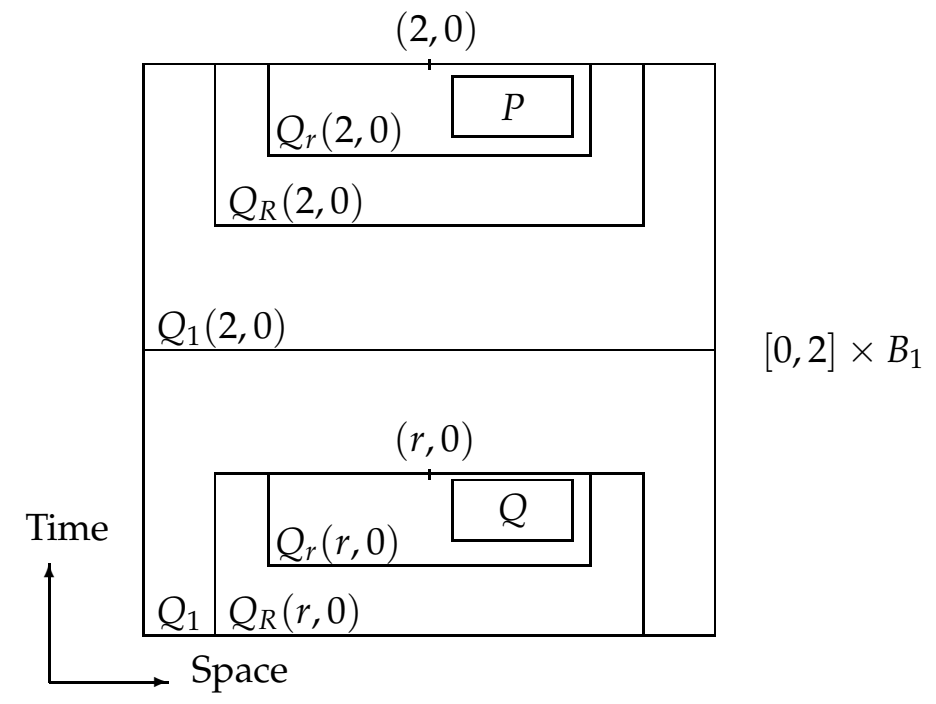

FIGURE 5. Relative positions of the sets used in the proof.

Fix any $\epsilon>0$ and let $\alpha_{\epsilon}$ and $K_{\epsilon}$ be the constants in PROPOSITION 2.5 with $R$ in place of $t$ there. For $\mu>0$, we write

$$
v_{\mu}=(u+\mu)^{-1}, f^{\mu}\left(t, x, v_{\mu} ; \omega\right):=f(t, x, u ; \omega) v_{\mu}^{2}, g_{i}^{\mu}\left(t, x, v_{\mu} ; \omega\right):=g_{i}(t, x, u ; \omega) v_{\mu}^{2} .
$$

By a direct calculation, $v_{\mu}(t+1, x)$ is a sub-solution of (1.1) on $Q_{1}(1,0)$ with $f$ and $g_{i}$ replaced by $f^{\mu}$ and $g_{i}^{\mu}$. We note here that $\left|f^{\mu}(v)\right|+\left|g^{\mu}(v)\right|_{\ell^{2}} \leq \Lambda|v|$ still holds.

Applying Proposition 2.4 to $v_{\mu}(t+1, x)$ with $p=\alpha_{\epsilon}$ and $a$ replaced by $a^{-1}$, we can find a $\Gamma_{\epsilon}$ depending only on $n, l, \Lambda$ and $\epsilon$ such that for all $\Gamma \geq \Gamma_{\epsilon}$,

$$
\mathbb{P}\left\{\sup _{Q_{r}(2,0)} v_{\mu}>a^{-1}, \Gamma\left\|v_{\mu}\right\|_{\alpha_{\epsilon}, Q_{R}(2,0)} \leq a^{-1}\right\} \leq \exp \left\{-\Gamma^{\delta\left(\alpha_{\epsilon}\right)}\right\} .
$$

This is equivalent to

$$
\mathbb{P}\left\{\inf _{Q_{r}(2,0)}(u+\mu)<a, \Gamma\left\|(u+\mu)^{-1}\right\|_{\alpha_{\epsilon}, Q_{R}(2,0)} \leq a^{-1}\right\} \leq \exp \left\{-\Gamma^{\delta\left(\alpha_{\varepsilon}\right)}\right\} .
$$


At the same time, we have an obvious inequality

$$
\begin{aligned}
& \mathbb{P}\left\{\inf _{Q_{r}(2,0)}(u+\mu)<a,\|u+\mu\|_{\alpha_{\epsilon}, Q_{R}(r, 0)} \geq \Gamma K_{\epsilon} a\right\} \\
& \quad \leq \mathbb{P}\left\{\mathcal{F}\left[u+\mu, \alpha_{\epsilon}\right]_{Q_{R}(2,0), Q_{R}(r, 0)}^{1 / \alpha_{\epsilon}}>K_{\epsilon}\right\} \\
& \quad+\mathbb{P}\left\{\inf _{Q_{r}(2,0)}(u+\mu)<a,\|u+\mu\|_{\alpha_{\epsilon}, Q_{R}(r, 0)} \geq \mathcal{F}\left[u+\mu, \alpha_{\epsilon}\right]_{Q_{R}(2,0), Q_{R}(r, 0)}^{1 / \alpha_{\epsilon}} \Gamma a\right\} .
\end{aligned}
$$

The first term on the right hand side is bounded by $\epsilon$ by PROPOSITION 2.5 after taking $t=r$; the second term is equivalent to the left hand side of (2.3), thus it is bounded by $\epsilon$ if $\Gamma$ is sufficiently large. Therefore the last inequality gives,

$$
\mathbb{P}\left\{\inf _{Q_{r}(2,0)}(u+\mu)<a, \quad\|u+\mu\|_{\alpha_{\epsilon}, Q_{R}(r, 0)} \geq \Gamma K_{\epsilon} a\right\} \leq 2 \epsilon .
$$

Now we look at $Q_{R}(r, 0)$. On this rectangular region, $\bar{v}_{\mu}:=u+\mu$ is a solution of (1.1) with $f$ and $g_{i}$ replaced by $\bar{f}^{\mu}\left(t, x, \bar{v}_{\mu} ; \omega\right):=f(t, x, u ; \omega)$ and $\bar{g}_{i}^{\mu}\left(t, x, \bar{v}_{\mu} ; \omega\right):=g_{i}(t, x, u ; \omega)$. Applying PROPOSITION 2.4 again, we can get another constant $\Gamma_{\epsilon}^{\prime}$ depending only on $n, l, \Lambda$ and $\epsilon$ such that for all $\Gamma \geq \Gamma_{\epsilon}^{\prime}$ and $a>0$,

$$
\mathbb{P}\left\{\sup _{Q_{r}(r, 0)}(u+\mu)>a, \quad \Gamma\|u+\mu\|_{\alpha_{\epsilon}, Q_{R}(r, 0)} \leq a\right\} \leq 2 \epsilon .
$$

From (2.4), (2.5) and the obvious inequality

$$
\begin{aligned}
& \mathbb{P}\left\{\sup _{Q_{r}(r, 0)}(u+\mu)>a, \Gamma \inf _{Q_{r}(2,0)}(u+\mu)<a\right\} \\
& \leq \mathbb{P}\left\{\sup _{Q_{r}(r, 0)}(u+\mu)>a,\|u+\mu\|_{\alpha_{\epsilon}, Q_{R}(r, 0)} \leq a / \Gamma_{\epsilon}^{\prime}\right\} \\
&+\mathbb{P}\left\{\inf _{Q_{r}(2,0)}(u+\mu)<a / \Gamma,\|u+\mu\|_{\alpha_{\epsilon}, Q_{R}(r, 0)} \geq a / \Gamma_{\epsilon}^{\prime}\right\},
\end{aligned}
$$

we will have

$$
\mathbb{P}\left\{\sup _{Q_{r}(r, 0)}(u+\mu)>a, \Gamma \inf _{Q_{r}(2,0)}(u+\mu)<a\right\} \leq 4 \epsilon
$$

if we pick $\Gamma_{\epsilon}^{\prime}$ sufficiently large first and then let $\Gamma / \Gamma_{\epsilon}^{\prime}$ be sufficiently large.

The last inequality implies

$$
\mathbb{P}\left\{\sup _{Q_{r}(r, 0)}(u+\mu) \geq 2 a, \quad \Gamma \inf _{Q_{r}(2,0)}(u+\mu)<a\right\} \leq 4 \epsilon .
$$

Take $\mu=1 / m$ and let $m \rightarrow \infty$, we have from Fatou's lemma,

$$
\mathbb{P}\left\{\sup _{Q_{r}(r, 0)} u \geq 2 a, \Gamma \inf _{Q_{r}(2,0)} u<a\right\} \leq 4 \epsilon .
$$

This further leads to

$$
\mathbb{P}\left\{\sup _{Q_{r}(r, 0)} u>4 a, \Gamma \inf _{Q_{r}(2,0)} u \leq a / 2\right\} \leq 4 \epsilon,
$$

which implies the desired statement if we use $\epsilon / 4$ in place of $\epsilon$. 
Case II. The length of $I_{P}$ is different from that of $I_{Q}$.

Without loss of generality we assume $I_{P}$ is longer, we cover $P$ by finitely many $P_{i}$ 's of the exact same shape as $Q$. Applying the result from Case I to $P_{i}$ and $Q$ for all $i$, we have when $\Gamma$ is large

$$
\mathbb{P}\left\{\sup _{P_{i}} u>a, \quad \Gamma \inf _{Q} u \leq a\right\} \leq \epsilon .
$$

Therefore we have

$$
\mathbb{P}\left\{\sup _{P_{i}} u>a, \quad \Gamma \inf _{Q} u \leq a\right\} \leq C \epsilon,
$$

where $C$ is the number of rectangles used to cover $P$.

We now turn to the strict positivity result, which we restate here.

Theorem 2.7. Let $u$ be a solution of the SPDE (1.1) on $\mathbb{R}^{+} \times \mathbb{R}^{n}$ with a (deterministic) non-negative and not identically vanishing initial condition $u(0)=u_{0} \in L^{2}\left(\mathbb{R}^{n}\right)$. Then for probability one, $u(t, x)$ is positive for all $x \in \mathbb{R}^{n}$ and $t>0$.

Proof. We prove by contradiction. Suppose the strict positivity conclusion is false, then for some $t_{0}>0$, we have

$$
\mathbb{P}\left\{\forall x \in \mathbb{R}^{n}, \forall t \in\left[\frac{t_{0}}{2}, t_{0}\right] \mid u(t, x)>0\right\}<1 .
$$

We will first prove $u$ is non-negative. This is a well known result and the method of proof is to calculate $\mathbb{E}\left\|u^{-}\right\|_{2, \mathbb{R}^{n}}^{2}$ as in Pardoux [10]. By formally applying Itô's formula on $h(u)=\left|u^{-}\right|^{2}$, we have,

$$
\begin{aligned}
d\left\|u^{-}(t)\right\|_{2, \mathbb{R}^{n}}^{2}= & -2\left\langle\nabla u^{-}(t), \mathbb{A} \nabla u^{-}(t)\right\rangle d t+2\left\langle g_{i}(u), u^{-}(t)\right\rangle d w_{t}^{i} \\
& +\left[\int_{\mathbb{R}^{n}}\left\{|g(u(t))|^{2}+2 u^{-}(t) f(u(t))\right\} 1_{\left\{u^{-}(t)>0\right\}} d x\right] d t .
\end{aligned}
$$

The justification for the application of Itô's formula is the same as in [5, Remark 2.3].

Taking the expectation on both sides and noting the fact that $u_{0}$ is non-negative, we have

$$
\mathbb{E}\left\|u^{-}(s)\right\|_{2, \mathbb{R}^{n}}^{2} \leq \mathbb{E} \int_{0}^{s}\left[\int_{\mathbb{R}^{n}}\left\{|g(u(t))|^{2}+2 u^{-}(t) f(u(t))\right\} 1_{\left\{u^{-}(t)>0\right\}} d x\right] d t .
$$

Using the linear growth condition on $f$ and $g$ and Gronwall's inequality, we have $\mathbb{E}\left\|u^{-}(t)\right\|_{2, \mathbb{R}^{n}}^{2}=$ 0 for all $t$. This proves the non-negativity of $u$ after we recall from [5, Theorem 1.2] that $u$ is continuous in both time and space after time 0.

With this non-negativity result, (2.6) can be rewritten as

$$
\mathbb{P}\left\{\exists x \in \mathbb{R}^{n}, t \in\left[\frac{t_{0}}{2}, t_{0}\right] \mid u(t, x)=0\right\}>0 .
$$

Due to the continuity of $u$ after $t=0$, if we cover $\left[t_{0} / 2, t_{0}\right] \times \mathbb{R}^{n}$ by countably many copies of $\left[t_{0} / 2, t_{0}\right] \times\{x|| x \mid \leq 1\}$, there must be one of them, say $P$, satisfying

$$
\mathbb{P}\left\{\inf _{P} u=0\right\}>0
$$

At the same time, since the deterministic initial condition $u_{0}$ is not identically vanishing, there must be a small time after 0 where $\|u\|_{2, \mathbb{R}^{n}}$ stays positive (the time may vary among different $\omega^{\prime} \mathrm{s}$ ). 
Therefore if we cover $\left(0, t_{0} / 3\right] \times \mathbb{R}^{n}$ by countably many compact sets, there must be one of them, say $Q_{0}$, satisfying

$$
\mathbb{P}\left\{\inf _{P} u=0, \sup _{Q_{0}} u>0\right\}>0 .
$$

We now further choose an $a>0$ and a space-time domain $Q \subset\left(0,5 t_{0} / 12\right] \times \mathbb{R}^{n}$ slightly larger than $Q_{0}$ such that

$$
\mathbb{P}\left\{\inf _{P} u=0, \sup _{Q} u>a\right\}>0 .
$$

However, THEOREM 1.1 shows that we can find a large $\Gamma$ such that,

$$
\mathbb{P}\left\{\Gamma \inf _{P} u \leq a, \sup _{Q} u>a\right\}<\mathbb{P}\left\{\inf _{P} u=0, \sup _{Q} u>a\right\} .
$$

This gives a contradiction since the event on the right hand side implies the event on the left hand side.

\section{LOCAL PROPERTIES OF THE SUB-SOLUTION}

In our previous work [5], a stochastic variant of the classical De Giorgi's iteration has been developed for studying the global properties of solution of (1.1). In this section, we will adapt this method to prove local properties for the solution. In other words, we will prove PROPOSITION 2.3. We start with the following result.

Proposition 3.1. Let $\epsilon$ be a constant in $(0,1]$ and $u$ be a sub-solution to

$$
\partial_{t} u=\operatorname{div}(\mathbb{A} \nabla u)+\epsilon^{2} f(t, x, u ; \omega)+\epsilon\left(g_{i}(t, x, u ; \omega) d w_{t}^{i}\right)
$$

in $Q_{1}$. Then there exist $\Gamma(0)$ and $\delta(0)$ depending only on $n, \iota$ and $\Lambda$ such that for all $a>0$ and $\Gamma \geq \Gamma(0)$,

$$
\mathbb{P}\left\{\sup _{Q_{1 / 2}} u>a,(1 / 2)^{-(n+1) / 2}\|u\|_{4,2, Q_{1}} \leq a / \Gamma\right\} \leq \exp \left\{-\Gamma^{\delta(0)} / \epsilon^{2}\right\} .
$$

The proof of the proposition is a verbatim repetition of the proof for [5. Proposition 3.3] with minor adjustments.

We proceed as in [5]. We write $b_{k}=2^{-1}+2^{-k-1}$ and pick $I_{k}:=\left[1-b_{k}^{2}, 1\right]$, a sequence of time intervals shrinking from $[0,1]$ to $[3 / 4,1]$. We define a sequence of smooth non-negative cut-off functions $\varphi_{k}$ bounded by 1 such that $\varphi_{k}$ is 1 on $B_{b_{k}}$ and 0 outside of $B_{b_{k-1}}$ for $k \geq 1$ and $\varphi_{0} \equiv 1$ on $B_{1}$. We also require $\varphi_{k}$ to have a gradient globally bounded by $n 2^{k+2}$. For each $a>0$, we write $u_{k, a}=\left(u-a\left(1-2^{-k}\right)\right)^{+}$and let

$$
U_{k, a}:=\left\|u_{k, a} \varphi_{k}\right\|_{4,2, I_{k} \times B_{1}}^{2} .
$$

For simplicity we denote $f(t, x, u ; \omega)$ and $g_{i}(t, x, u ; \omega)$ by $f(u)$ and $g_{i}(u)$, respectively. Assume $n \geq 3$ for now. We have the following iterative inequality.

Proposition 3.2. There exist constants $C_{0}=C(n, l, \Lambda)$ and $\delta=\delta(n, l, \Lambda)$ such that for $a \geq 1$

$$
U_{k, a} \leq C_{0}^{k} a^{-2 \delta}\left(U_{k-1, a}+X_{k-1, a}^{*}\right) U_{k-1, a}^{\delta}
$$

where

$$
X_{k-1, a}^{*}=\epsilon \sup _{1-b_{k-1}^{2} \leq s \leq t \leq 1} \int_{s}^{t}\left\langle g_{i}(u(\tau)), u_{k, a}(\tau) \varphi_{k}^{2}\right\rangle d w_{\tau}^{i} .
$$


Proof. During this proof, the constant $C$ is enlarged from line to line as we proceed.

We note that $\left\|u_{k, a}(t) \varphi_{k}\right\|_{2, B_{b_{k-1}}}^{2}=\left\|u_{k, a}(t) \varphi_{k}\right\|_{2, B_{1}}^{2}$. Hölder's inequality with the conjugate exponents $(n+1) / n$ and $n+1$ gives

$$
\left\|u_{k, a}(t) \varphi_{k}\right\|_{2, B_{b_{k-1}}}^{2} \leq\left\|u_{k, a}(t) \varphi_{k}\right\|_{2(n+1) / n, B_{b_{k-1}}}^{2} \cdot\left|\left\{u_{k, a}(t)>0\right\} \cap B_{b_{k-1}}\right|^{1 /(n+1)} .
$$

Using Chebyshev's inequality, we have

$$
\left|\left\{u_{k, a}(t)>0\right\} \cap B_{b_{k-1}}\right|=\left|\left\{u_{k-1, a}(t)>2^{-k} a\right\} \cap B_{b_{k-1}}\right| \leq\left(\frac{2^{k}}{a}\right)^{2}\left\|u_{k-1, a}(t)\right\|_{2, B_{b_{k-1}}}^{2} .
$$

Noting $\left\|u_{k-1, a}(t)\right\|_{2, B_{b_{k-1}}}^{2} \leq\left\|u_{k-1, a}(t) \varphi_{k-1}\right\|_{2, B_{1}}^{2}$, squaring (3.5) and integrating with respect to $t$ on $I_{k}$ we have

$$
U_{k, a}^{2} \leq\left(\frac{2^{k}}{a}\right)^{4 /(n+1)} \int_{I_{k}}\left\|u_{k, a}(t) \varphi_{k}\right\|_{2(n+1) / n, B_{b_{k-1}}}^{4}\left\|u_{k-1, a}(t) \varphi_{k-1}\right\|_{2, B_{1}}^{4 /(n+1)} d t .
$$

Applying Hölder's inequality again with the same conjugate exponents, we obtain

$$
\begin{aligned}
U_{k, a} \leq\left(\frac{2^{k}}{a}\right)^{2 /(n+1)} & \times\left(\int_{I_{k}}\left\|u_{k, a}(t) \varphi_{k}\right\|_{2(n+1) / n, B_{b_{k-1}}}^{4(n+1) / n} d t\right)^{n / 2(n+1)} \\
& \times\left(\int_{I_{k}}\left\|u_{k-1, a}(t) \varphi_{k-1}\right\|_{2, B_{1}}^{4} d t\right)^{1 / 2(n+1)} .
\end{aligned}
$$

The second factor is $\left\|u_{k, a} \varphi_{k}\right\|_{4(n+1) / n, 2(n+1) / n, I_{k} \times B_{b_{k-1}}}^{2}$, and the $L_{t}^{p} L_{x}^{q}$ interpolation inequality [5, Proof of Proposition 2.1] leads to

$$
\left\|u_{k, a} \varphi_{k}\right\|_{4(n+1) / n, 2(n+1) / n, I_{k} \times B_{b_{k-1}}}^{2} \leq \sup _{t \in I_{k}}\left\|u_{k, a}(t) \varphi_{k}\right\|_{2, B_{b_{k-1}}}^{2}+\int_{I_{k}}\left\|u_{k, a}(t) \varphi_{k}\right\|_{2 n /(n-2), B_{b_{k-1}}}^{2} d t
$$

Applying the Sobolev inequality on $B_{1}$ to the second term on the right side of the above inequality and then substituting the result in (3.6), we obtain

$$
U_{k, a} \leq C\left(\frac{2^{k}}{a}\right)^{2 /(n+1)}\left[\sup _{t \in I_{k}}\left\|u_{k, a}(t) \varphi_{k}\right\|_{2, B_{b_{k-1}}}^{2}+\int_{I_{k}}\left\|\nabla\left(u_{k, a}(t) \varphi_{k}\right)\right\|_{2, B_{b_{k-1}}}^{2} d t\right] U_{k-1, a}^{1 /(n+1)} .
$$

after noting the fact that the third factor on the right side of (3.6) is bounded by $U_{k-1, a}^{1 /(n+1)}$.

We now try to bound the right-hand side of (3.7). For the same reasoning as in [5, Remark 2.3], Itô's formula can be applied to the composition $h_{k}(u(t)):=\left|u_{k, a}(t) \varphi_{k}\right|^{2}$,

$$
\begin{aligned}
d\left\|u_{k, a}(t) \varphi_{k}\right\|_{2, B_{b_{k-1}}}^{2} & =-2\left\langle\nabla u_{k, a}(t), \mathbb{A} \nabla\left(u_{k, a}(t) \varphi_{k}^{2}\right)\right\rangle d t+2\left\langle\epsilon g_{i}(u), u_{k, a}(t) \varphi_{k}^{2}\right\rangle d w_{t}^{i} \\
& +\left[\int_{B_{b_{k-1}}}\left\{\left|\epsilon g(u(t)) \varphi_{k}\right|^{2}+2 u_{k, a}(t) \varphi_{k}^{2} \epsilon^{2} f(u(t))\right\} 1_{\left\{u_{k, a}(t)>0\right\}} d x\right] d t
\end{aligned}
$$


For the first term on the right-hand side, by using the uniform ellipticity of $A$ and the bounds on $\varphi$ and $\nabla \varphi_{k}$, we have

$$
\begin{aligned}
\left\langle\nabla u_{k, a}, \mathbb{A} \nabla\left(u_{k, a}(t) \varphi_{k}^{2}\right)\right\rangle & =\left\langle\nabla u_{k, a}, \mathbb{A} \varphi_{k}^{2} \nabla u_{k, a}(t)\right\rangle+2\left\langle\nabla u_{k, a}, \mathbb{A} u_{k, a}(t) \varphi_{k} \nabla \varphi_{k}\right\rangle \\
& \geq \iota\left\|\varphi_{k} \nabla u_{k, a}\right\|_{2, B_{1-b_{k-1}}}^{2}-\frac{\iota}{2}\left\|\varphi_{k} \nabla u_{k, a}\right\|_{2, B_{b_{k-1}}}^{2}-C\left\|u_{k, a} \nabla \varphi_{k}\right\|_{2, B_{b_{k-1}}}^{2} \\
& \geq \frac{\iota}{2}\left\|\varphi_{k} \nabla u_{k, a}\right\|_{2, B_{b_{k-1}}}^{2}-C^{k}\left\|u_{k, a}\right\|_{2, B_{b_{k-1}}}^{2} \\
& \geq \frac{l}{2}\left\|\varphi_{k} \nabla u_{k, a}\right\|_{2, B_{b_{k-1}}}^{2}-C^{k}\left\|u_{k-1, a} \varphi_{k-1}\right\|_{2, B_{1}}^{2} .
\end{aligned}
$$

For the third term on the right-hand side of (3.8), we observe that if $u_{k, a}>0$, then $0<a \leq 2^{k} u_{k-1, a}$ and $0<u \leq u_{k-1, a}+a \leq\left(1+2^{k}\right) u_{k-1, a}$. By the linear growth assumption on $f$ and $g$, the third term is bounded by $C^{k}\left\|u_{k-1, a} \varphi_{k-1}\right\|_{2, B_{1}}^{2} d t$. Now, integrating (3.8) from $t^{\prime}$ to $t$ with $t^{\prime} \in I_{k-1} \backslash I_{k}$ and $t \in I_{k}$ and applying Cauchy-Schwartz inequality on the integral of $\left\|u_{k-1, a} \varphi_{k-1}\right\|_{2, B_{1}}^{2}$ gives

$$
\begin{aligned}
\left\|u_{k, a}(t) \varphi_{k}\right\|_{2, B_{b_{k-1}}}^{2}+ & \frac{\iota}{2} \int_{t^{\prime}}^{t}\left\|\varphi_{k} \nabla u_{k, a}(s)\right\|_{2, B_{b_{k-1}}}^{2} d s \\
& \leq\left\|\varphi_{k} u_{k, a}\left(t^{\prime}\right)\right\|_{2, B_{b_{k-1}}}^{2}+C^{k} U_{k-1, a}+2 \epsilon \int_{t^{\prime}}^{t}\left\langle g_{i}(u(s)), u_{k, a}(s) \varphi_{k}^{2}\right\rangle d w_{s}^{i} .
\end{aligned}
$$

Taking supremum over $t \in I_{k}$, we have

$$
\begin{aligned}
\sup _{t \in I_{k}}\left\|u_{k, a}(t) \varphi_{k}\right\|_{2, B_{1-b_{k-1}}}^{2} & +\int_{t_{0}}^{1}\left\|\varphi_{k} \nabla u_{k, a}(s)\right\|_{2, B_{1-b_{k-1}}}^{2} d s \\
& \leq C\left\|u_{k, a}\left(t^{\prime}\right) \varphi_{k}\right\|_{2, B_{1-b_{k-1}}}^{2}+C^{k} U_{k-1, a}+C X_{k-1, a}^{*}
\end{aligned}
$$

with $X_{k-1, \alpha}^{*}$ defined in (3.4). Integrating (3.9) on $I_{k-1} \backslash I_{k}$ with respect to $t^{\prime}$, combining the resulting inequality, (3.7) and the fact that

$$
\begin{aligned}
\int_{I_{k}}\left\|\nabla\left(u_{k, a}(t) \varphi_{k}\right)\right\|_{2, B_{b_{k-1}}}^{2} d t & \leq C \int_{I_{k}}\left\|\varphi_{k} \nabla u_{k, a}(t)\right\|_{2, B_{b_{k-1}}}^{2} d t+C \int_{I_{k}}\left\|u_{k, a}(t) \nabla \varphi_{k}\right\|_{2, B_{b_{k-1}}}^{2} d t \\
& \leq C \int_{I_{k}}\left\|\varphi_{k} \nabla u_{k, a}(t)\right\|_{2, B_{b_{k-1}}}^{2} d t+C^{k} \int_{I_{k}}\left\|u_{k-1, a}(t)\right\|_{2, B_{b_{k-1}}}^{2} d t \\
& \leq C \int_{I_{k}}\left\|\varphi_{k} \nabla u_{k, a}(t)\right\|_{2, B_{b_{k-1}}}^{2} d t+C^{k} U_{k-1},
\end{aligned}
$$

we obtain the desired iterative inequality (3.3).

Remark 3.3. When $n=1$ or 2 , due to the different form of Sobolev inequality in those dimensions, we need to substitute the $1 /(n+1)$ in the proof by some positive number $\delta$ between 0 and $1 / 3$ and adjust the conjugate coefficients in the inequalities accordingly.

We are ready to proceed to the next step, namely, comparing $X_{k, a}^{*}$ and $U_{k, a}$.

Consider the continuous martingale for any fixed $a>0$,

$$
X_{k, t}:=\epsilon \int_{0}^{t}\left\langle g_{i}(u(s)), u_{k+1, a}(s) \varphi_{k+1}^{2}\right\rangle d w_{s}^{i}
$$

and recall from (3.4) that $X_{k, a}^{*}=\sup _{1-b_{k}^{2} \leq s \leq t \leq 1}\left(X_{k, t}-X_{k, s}\right)$.

Lemma 3.4. There exists a constant $C=C(n, l, \Lambda)$ such that for all positive $\alpha, \beta$ and $k$,

$$
\mathbb{P}\left\{X_{k, a}^{*} \geq \alpha \beta, U_{k, a} \leq \beta\right\} \leq C \exp \left\{-\alpha^{2} /\left(C^{k} \epsilon^{2}\right)\right\} .
$$


Proof. We use $\left\langle X_{k}\right\rangle$ to denote the quadratic variation process of $X_{k, t}$. If we can show that there is a constant $C$ such that

$$
\left\langle X_{k}\right\rangle_{1}-\left\langle X_{k}\right\rangle_{1-b_{k}^{2}} \leq \epsilon^{2} C^{k} U_{k, a}^{2}
$$

then

$$
\left\{X_{k, a}^{*} \geq \alpha \beta, U_{k a} \leq \beta\right\} \subset\left\{\sup _{1-b_{k}^{2} \leq s \leq t \leq 1}\left(X_{k, t}-X_{k, s}\right) \geq \alpha \beta,\left\langle X_{k}\right\rangle_{1}-\left\langle X_{k}\right\rangle_{1-b_{k}^{2}} \leq \epsilon^{2} C^{k} \beta^{2}\right\}
$$

and the desired estimate follows immediately from the fact that $X_{k, t}$ is a time-changed Brownian motion and the corresponding estimate for Brownian motion, see [5, Lemma 3.1] for details. To prove (3.10), we start with

$$
\left\langle X_{k}\right\rangle_{1}-\left\langle X_{k}\right\rangle_{1-b_{k}^{2}}=\epsilon^{2} \sum_{i \in \mathbb{N}} \int_{I_{k}}\left\langle g_{i}(u), u_{k+1} \varphi_{k+1}^{2}\right\rangle^{2} d s,
$$

which follows from the definition of $X_{k, t}$. We observe that if $u_{k+1, a}>0$, then $0<a \leq 2^{k+1} u_{k, a}$ and $0<u \leq u_{k, a}+a \leq\left(1+2^{k+1}\right) u_{k, a}$. By Minkowski's inequality (integral form) and the linear growth assumption on $g$ we have

$$
\sum_{i \in \mathbb{N}}\left(\int_{\mathbb{R}^{n}} g_{i}(u) u_{k+1, a} \varphi_{k+1}^{2} d x\right)^{2} \leq\left(\int_{\mathbb{R}^{n}}|g(u)| u_{k+1, a} \varphi_{k+1}^{2} d x\right)^{2} \leq C^{k}\left(\int_{\mathbb{R}^{n}} u_{k, a}^{2} \varphi_{k}^{2} d x\right)^{2} .
$$

Integrating over the interval $I_{k}$ we obtain the desired inequality (3.10).

With LEMma 3.4, we can now use a Borel-Cantalli argument to prove Proposition 3.1 . Proof of PROPOSITION 3.1 We start with the observation that $\left\{\left\|u^{+}\right\|_{\infty, Q_{1 / 2}}>a\right\} \subset G_{a}^{c}$, where $G_{a}=$ $\left\{\lim _{k \rightarrow \infty} U_{k, a}=0\right\}$. Consider the events $\mathcal{E}_{k}=\left\{U_{k, a} \leq(a / \Gamma)^{2} \gamma^{k}\right\}$ for a constant $\gamma<1$ to be determined later. Since $\|u\|_{4,2, Q_{1}}=\sqrt{U_{0, a}}$, it suffices to prove

$$
\mathbb{P}\left\{G_{a}^{c} \cap \mathcal{E}_{0}\right\} \leq \exp \left\{-\Gamma^{\delta} / \epsilon^{2}\right\} .
$$

It is clear that

which implies

$$
G_{a}^{c} \subset \bigcup_{k \geq 0} \mathcal{E}_{k}^{c} \subset \mathcal{E}_{0}^{c} \cup\left[\bigcup_{k \geq 1}\left(\mathcal{E}_{k}^{c} \cap \mathcal{E}_{k-1}\right)\right]
$$

$$
\mathbb{P}\left\{G_{a}^{c} \cap \mathcal{E}_{0}\right\} \leq \sum_{k \geq 1} \mathbb{P}\left\{\mathcal{E}_{k}^{c} \cap \mathcal{E}_{k-1}\right\} .
$$

We estimate the probability $\mathbb{P}\left\{\mathcal{E}_{k}^{c} \cap \mathcal{E}_{k-1}\right\}$.

Let $\alpha=(2 C)^{k / 2} \Gamma^{\delta}$ and $\beta=a^{2} \gamma^{k-1} \Gamma^{-2}$ in LEMMA 3.4. If $X_{k-1, a}^{*} \leq \alpha \beta$ and $U_{k-1, a} \leq \beta$, then by the iterative inequality (3.3) in PROPOSITION 3.2 we have (after canceling $a^{2 \delta}$ !)

$$
U_{k, a} \leq \frac{C_{0}^{k}}{a^{2 \delta}}(\beta+\alpha \beta) \beta^{\delta}=\frac{\left(C_{0} \gamma^{\delta}\right)^{k}\left(1+(2 C)^{k / 2} \Gamma^{\delta}\right)}{\gamma^{1+\delta} \Gamma^{2 \delta}} \cdot \gamma \beta \leq \gamma \beta
$$

The last inequality holds if we choose $\gamma$ sufficiently small such that $\left(C_{1} \gamma^{\delta}\right)^{k}\left(1+(2 C)^{k / 2} \Gamma^{\delta}\right) \leq \Gamma^{\delta}$ for all $k \geq 1$ and $\Gamma \geq 1$ and then $\Gamma$ sufficiently large such that $\gamma^{1+\delta} \Gamma^{\delta} \geq 1$.

Now the above inequality implies that $\mathcal{E}_{k}^{c} \cap \mathcal{E}_{k-1} \subset\left\{X_{k-1, a}^{*}>\alpha \beta, U_{k-1, a} \leq \beta\right\}$. Its probability is estimated by LEMMA 3.4 and we have

$$
\mathbb{P}\left\{\mathcal{E}_{k}^{c} \cap \mathcal{E}_{k-1}\right\} \leq C \exp \left\{-\alpha^{2} /\left(C^{k} \epsilon^{2}\right)\right\}=C \exp \left\{-2^{k} \Gamma^{2 \delta} / \epsilon^{2}\right\}
$$


Using this in (3.11) we obtain, again for sufficiently large $\Gamma$,

$$
\mathbb{P}\left\{G_{a}^{c} \cap \mathcal{E}_{0}^{c}\right\} \leq C \sum_{k=1}^{\infty} \exp \left\{-2^{k} \Gamma^{2 \delta} / \epsilon^{2}\right\} \leq \exp \left\{-\Gamma^{\delta} / \epsilon^{2}\right\} .
$$

By lowering $\delta$ a little bit, we have proved (3.2) for all $a>0$.

Remark 3.5. The statements proved so far in this section do not require the initial condition of $u$ to be deterministic.

At this moment, we are just one step away from Proposition 2.3. For the ease of reference, we restate our goal here.

Proposition 3.6. Let $u$ be a sub-solution to (1.1) in $Q_{1}$. Then there exist $\Gamma(0)$ and $\delta(0)$ depending only on $n$, l and $\Lambda$ such that for all $a>0, r \in(0,1]$, and $\Gamma \geq \Gamma(0)$,

$$
\mathbb{P}\left\{\|u\|_{\infty, Q_{r / 2}}>a, \Gamma(r / 2)^{-(n+1) / 2}\|u\|_{4,2, Q_{r}} \leq a\right\} \leq \exp \left\{-\Gamma^{\delta(0)} / r^{2}\right\} .
$$

Proof. For any $r \in(0,1]$, we write $u_{r}(t, x ; \omega):=u\left(r^{2} t, r x ; \omega\right)$. By a direct calculation, $u_{r}$ satisfies equation

$$
\partial_{t} u_{r}(t, x)=\operatorname{div}\left(A \nabla u_{r}(t, x)\right)+r^{2} f\left(r^{2} t, r x, u_{r}(t, x) ; \omega\right)+r g_{i}\left(r^{2} t, r x, u_{r}(t, x) ; \omega\right) \dot{w}_{t}^{i},
$$

therefore (3.2) gives the desired result with supremum in place of $\|\cdot\|_{\infty}$. By considering $-u$ instead of $u$, we have our desired inequality.

Remark 3.7. As can be observed from the proof, for a fixed $r \leq 1$, the inequality (3.12) will hold as long as $u$ is a sub-solution of (1.1) on $Q_{r}$.

\section{FROM $\|\cdot\|_{4,2,}$ TO $\|\cdot\|_{p}$,}

We have controlled the tail of $\|u\|_{\infty, Q_{r / 2}}$ by the distribution of $\|u\|_{4,2, Q_{r}}$ in the last section. We now improve the control by lowering the 4,2-norm to any small $p$-norm in this section. We will prove PROPOSITION 2.4, which we restate here.

Proposition 4.1. Let $u$ be a sub-solution to (1.1) in $Q_{1}$. For every $2 \geq p>0$, there exist constants $\Gamma(p)$ and $\delta(p)$ depending only on $n, l, \Lambda$ and $p$ such that for all $a>0, \Gamma \geq \Gamma(p)$ and $0<r<R \leq 1$,

$$
\mathbb{P}\left\{\|u\|_{\infty, Q_{r}}>a,(R-r)^{-(n+2) / p}\|u\|_{p, Q_{R}} \leq a / \Gamma\right\} \leq \exp \left\{-\Gamma^{\delta(p)} / R^{2}\right\} .
$$

In fact, we will prove a more general result.

Proposition 4.2. Let $u$ be a sub-solution to (1.1) in $Q_{1}$. For all $4 \geq p>0$ and $2 \geq q>0$, there exist constants $\Gamma(p, q)$ and $\delta(p, q)$ depending only on $n, l, \Lambda$ and the pair $(p, q)$ such that for all $a>0$, $\Gamma \geq \Gamma(p, q)$ and $0<r<R \leq 1$,

$$
\mathbb{P}\left\{\|u\|_{\infty, Q_{r}}>a,(R-r)^{-(n / q+2 / p)}\|u\|_{p, q, Q_{R}} \leq a / \Gamma\right\} \leq \exp \left\{-\Gamma^{\delta(p, q)} / R^{2}\right\} .
$$

We will prove the above proposition as following. We will first strengthen PROPOSITION 3.6 by a covering argument, and then introduce two lemmas whose proofs will be postponed to the next section. After these, we will use the strengthened result as a starting point and repeatedly apply the two lemmas to prove PROPOSITION 4.2.

Our strengthen version of PROPOSITION 3.6 takes the following form. 
Proposition 4.3. Let $u$ be a sub-solution to (1.1) in $Q_{1}$, then there exist $\Gamma^{\prime}(0), \delta^{\prime}(0)$ depending on only $n, l$, and $\Lambda$ such that for every $a>0, \Gamma \geq \Gamma^{\prime}(0)$ and $0<r<R \leq 1$,

$$
\mathbb{P}\left\{\|u\|_{\infty, Q_{r}}>a,(R-r)^{-(n+1) / 2}\|u\|_{4,2, Q_{R}} \leq a / \Gamma\right\} \leq \exp \left\{\Gamma^{\delta^{\prime}(0)} / R^{2}\right\} .
$$

Proof. From (3.12), we have for all $0<R \leq 1$,

$$
\mathbb{P}\left\{\|u\|_{\infty, Q_{R / 2}}>a,(R / 2)^{-(n+1) / 2}\|u\|_{4,2, Q_{R}} \leq a / \Gamma\right\} \leq \exp \left\{-\Gamma^{\delta(0)} / R^{2}\right\} .
$$

We write $\theta=r / R \in(0,1)$ and consider the case $\theta \leq 1 / 2$ first. We note that $Q_{r} \subset Q_{R / 2}$ in this scenario. The last inequality implies

$$
\mathbb{P}\left\{\|u\|_{\infty, Q_{r}}>a, 2^{(n+1) / 2}(R-r)^{-(n+1) / 2}\|u\|_{4,2, Q_{R}} \leq a / \Gamma\right\} \leq \exp \left\{-\Gamma^{\delta(0)} / R^{2}\right\} .
$$

This leads to (4.1) for $r \leq \frac{1}{2} R$ with some $\delta^{\prime}(0)<\delta(0)$ and $\Gamma^{\prime}(0)>\Gamma(0)$.

For the case $\theta>1 / 2$, we have $Q_{(1-\theta) R}\left(t_{0}, x_{0}\right) \subset Q_{R}$ for every $\left(t_{0}, x_{0}\right) \in Q_{\theta R}$, it follows from PROPOSITION 3.6 applied to $u\left(t-1+t_{0}, x-x_{0}\right)$,

$$
\begin{aligned}
& \mathbb{P}\left\{\|u\|_{\infty, Q_{(1-\theta) R / 2}\left(t_{0}, x_{0}\right)}>a,((1-\theta) R / 2)^{-(n+1) / 2}\|u\|_{4,2, Q_{(1-\theta) R}\left(t_{0}, x_{0}\right)} \leq a / \Gamma\right\} \\
& \leq \exp \left\{-\Gamma^{\delta(0)} /((1-\theta) R)^{2}\right\} .
\end{aligned}
$$

Since $Q_{(1-\theta) R}\left(t_{0}, x_{0}\right) \subset Q_{R}$, this inequality implies $\mathbb{P}\left\{\|u\|_{\infty, Q_{(1-\theta) R / 2}\left(t_{0}, x_{0}\right)}>a,((1-\theta) R / 2)^{-(n+1) / 2}\|u\|_{4,2, Q_{R}} \leq a / \Gamma\right\} \leq \exp \left\{-\Gamma^{\delta(0)} /((1-\theta) R)^{2}\right\}$. Now there exists a constant $L$ depending only on the dimension $n$ such that we can choose $\lceil L(1-$ $\left.\theta)^{-(n+2)}\right\rceil$ points $\left(t_{i}, x_{i}\right)$ in $Q_{\theta R}$ satisfying $Q_{\theta R} \subset \bigcup_{i=1}^{\left\lceil L(1-\theta)^{-(n+2)}\right\rceil} Q_{(1-\theta) R / 2}\left(t_{i}, x_{i}\right)$. This implies that the event $\left\{\|u\|_{\infty, Q_{\theta R}}>a\right\}$ is contained in $\bigcup_{i=1}^{\left\lceil L(1-\theta)^{-(n+2)}\right\rceil}\left\{\|u\|_{\infty, Q_{(1-\theta) R / 2}\left(t_{i}, x_{i}\right)}>a\right\}$. Therefore we obtain,

$$
\begin{aligned}
\mathbb{P} & \left\{\|u\|_{\infty, Q_{\theta R}}>a,((1-\theta) R)^{-(n+1) / 2}\|u\|_{4,2, Q_{R}} \leq a / \Gamma\right\} \\
& \leq \sum_{i=1}^{\left\lceil L(1-\theta)^{-(n+2)}\right\rceil} \mathbb{P}\left\{\|u\|_{\infty, Q_{(1-\theta) R / 2}\left(t_{i}, x_{i}\right)}>a,((1-\theta) R)^{-(n+1) / 2}\|u\|_{4,2, Q_{R}} \leq a / \Gamma\right\} \\
& \leq 2 L(1-\theta)^{-(n+2)} \exp \left\{-2^{-(n+1) / 2} \Gamma^{\delta(0)} /((1-\theta) R)^{2}\right\} \\
& =\exp \left\{-\frac{\Gamma^{\delta(0)}}{R^{2}} \frac{2^{-(n+1) / 2}}{(1-\theta)^{2}}+(n+2) \log \frac{1}{1-\theta}+\log (2 L)\right\} .
\end{aligned}
$$

Combining the last inequality, (4.2), and the fact that

$$
-\frac{\Gamma^{\delta(0)}}{R^{2}} \frac{2^{-(n+1) / 2}}{(1-\theta)^{2}}+(n+2) \log \frac{1}{1-\theta}+\log (2 L)<-2^{-(n+1) / 2} \frac{\Gamma^{\delta(0)}}{R^{2}}, \forall \theta \in\left(\frac{1}{2}, 1\right), R \in(0,1]
$$

when $\Gamma$ is large, we obtain PROPOSITION 4.3 by shrinking $\delta^{\prime}(0)$ and enlarging $\Gamma^{\prime}(0)$.

We now introduce the following two lemmas which will be proved later in SECTION 5. 


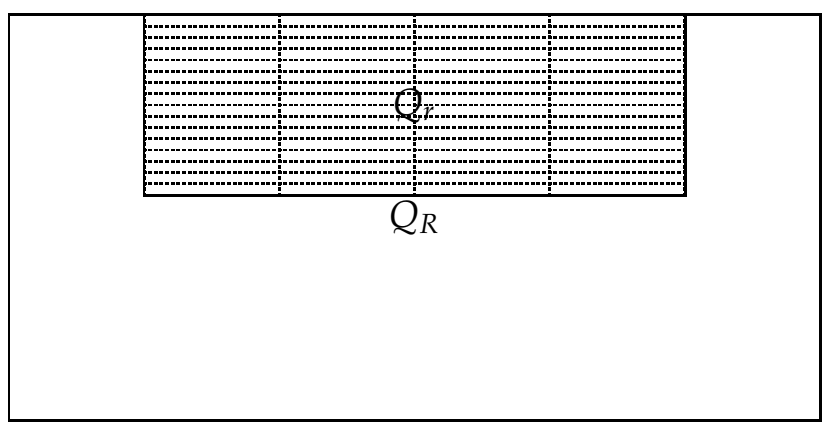

FIGURE 6. An example of the covering used when $\theta=\frac{2}{3}$

Lemma 4.4 (Exponent Reduction: Time). Let $u$ be a random function in $Q_{1}$. Suppose that for some $\alpha$ and $q, u \in L^{\alpha}\left([0,1], L^{q}\left(B_{1}\right)\right)$ almost surely. Suppose further that there exist two constants $\delta(\alpha)>0$ and $\Gamma(\alpha)$ such that for all $0<r<R \leq 1, a>0$ and $\Gamma>\Gamma(\alpha)$,

$$
\mathbb{P}\left\{\|u\|_{\infty, Q_{r}}>a,(R-r)^{-(n / q+2 / \alpha)}\|u\|_{\alpha, q, Q_{R}} \leq a / \Gamma\right\} \leq \exp \left\{-\Gamma^{\delta(\alpha)} / R^{2}\right\} .
$$

Then for any $\beta \in(\alpha / 2, \alpha)$, there exist $\delta(\beta)>0$ and $\Gamma(\beta)$ depending only on $n, \delta(\alpha)$ and $\Gamma(\alpha)$ such that

$$
\mathbb{P}\left\{\|u\|_{\infty, Q_{r}}>a, r^{-(n / q+2 / \beta)}\|u\|_{\beta, q, Q_{2 r}} \leq a / \Gamma\right\} \leq \exp \left\{-\Gamma^{\delta(\beta)} / 4 r^{2}\right\},
$$

for all $\Gamma>\Gamma(\beta), a>0$ and $0<r \leq 1 / 2$.

Lemma 4.5 (Exponent Reduction: Space). Let $u$ be a random function in $Q_{1}$. Suppose that for some $p$ and $\alpha, u \in L^{p}\left([0,1], L^{\alpha}\left(B_{1}\right)\right)$ almost surely. Suppose further that there exist two constants $\delta(\alpha)>0$ and $\Gamma(\alpha)$ such that for all $0<r<R \leq 1, a>0$ and $\Gamma>\Gamma(\alpha)$,

$$
\mathbb{P}\left\{\|u\|_{\infty, Q_{r}}>a,(R-r)^{-(n / \alpha+2 / p)}\|u\|_{p, \alpha, Q_{R}} \leq a / \Gamma\right\} \leq \exp \left\{-\Gamma^{\delta(\alpha)} / R^{2}\right\} .
$$

Then for any $\beta \in(\alpha / 2, \alpha)$, there exist $\delta(\beta)>0$ and $\Gamma(\beta)$ depending only on $n, \delta(\alpha)$ and $\Gamma(\alpha)$ such that

$$
\mathbb{P}\left\{\|u\|_{\infty, Q_{r}}>a, r^{-(n / \beta+2 / p)}\|u\|_{p, \beta, Q_{2 r}} \leq a / \Gamma\right\} \leq \exp \left\{-\Gamma^{\delta(\beta)} / 4 r^{2}\right\},
$$

for all $\Gamma>\Gamma(\beta), a>0$ and $0<r \leq 1 / 2$.

To finish the proof of PROPOSITION 4.2, we strengthen the conclusion of the two lemmas above, namely, (4.4) and (4.6) to the forms of (4.3) and (4.5) with the same covering argument as in the proof of PROPOSITION 4.3. From here, PROPOSITION 4.3 can be viewed as a starting point and PROPOSITION 4.2 is obtained by repeatedly applying LEMMA 4.4 and LEMMA 4.5.

\section{Proof of the Exponent Reduction Lemma}

In this section, we prove LEMMA 4.4 and LEMMA 4.5. The two proofs are almost completely identical, they both come from combining an iterative method used by Fanghua Lin in [4] and the Borel-Cantelli type argument used in [5, Proposition 3.3]; see [4, Chapter 4] for a detailed exposition in the classical case. 
We will only prove LEMMA 4.4 in detail and point out the differences for LEMMA 4.5. We start with an auxiliary parameter $\tau \in(0,1)$ which will be determined later. Let $r_{0}=r$ and $r_{i+1}=$ $r_{i}+r(1-\tau) \tau^{i}$ be a sequence of numbers increasing from $r$ to $2 r$.

We will repeatedly use (4.3) on each pair of sets $\left(Q_{r_{i}}, Q_{r_{i+1}}\right)$ and then sum up the inequalities. However, the original form of (4.3) is not fit for estimation after summation since the variable $a$ in the inequality has to be a constant. To circumvent such issue, we introduce the following probabilistic lemma.

Lemma 5.1. Assume $X, Y$ and $Z$ are three non-negative random variables with $Y \geq K Z$ for some $K>0$, suppose that there exist some $N_{0}, g$ and $\delta>0$ such that for all $b>0$ and $N>N_{0}$,

$$
\mathbb{P}\{X>b, Y N \leq b\} \leq \exp \left\{-g N^{\delta}\right\}
$$

Then for all $b>0$ and $N$ such that $K N^{2} /(K N+1)>N_{0}$,

$$
\mathbb{P}\{X+Z>b, Y N+Z \leq b\} \leq \exp \left\{-g\left(K N^{2} /(K N+1)\right)^{\delta}\right\} .
$$

Proof. We show the following inclusion

$$
\{X+Z>b, Y N+Z \leq b\} \subseteq\{X>K N b /(K N+1), Y N \leq b\} .
$$

(5.2) follows immediately from this and (5.1).

To prove the inclusion, we note that $Y \geq K Z \geq 0$ and $Y N+Z \leq b$ imply $Y N \leq b$ and $(K N+$ $1) Z \leq b$. The second inequality and $X+Z>b$ then imply $X>b-Z \geq K N b /(K N+1)$.

With LEMMA 5.1 in hand, we can now start the proof of LEMMA 4.4.

Proof of LEMMA 4.4. We will first assume $r<1 / 2$. We write $\gamma=-1+\alpha / \beta<1, \lambda=n / q+2 / \alpha$ and denote the volume of $B_{1}$ by $V$. We introduce an inequality which will play a key role in the proof of the lemma. From the $L_{t}^{p} L_{x}^{q}$ interpolation inequality and Hölder's inequality, with some constant $C_{\alpha, \beta}$ we have for all $\epsilon>0$ and $l>0$,

$$
\begin{aligned}
\|u\|_{\alpha, q, Q_{l}} & \leq\|u\|_{\infty, q, Q_{l}}^{1-\beta / \alpha}\|u\|_{\beta, q, Q_{l}}^{\beta / \alpha} \\
& \leq \epsilon\|u\|_{\infty, q, Q_{l}}+C_{\alpha, \beta} \epsilon^{-\gamma}\|u\|_{\beta, q, Q_{l}} \\
& \leq \epsilon V^{1 / q} l^{n / q}\|u\|_{\infty, Q_{l}}+C_{\alpha, \beta} \epsilon^{-\gamma}\|u\|_{\beta, q, Q_{l}} .
\end{aligned}
$$

For simplicity, we will use the following notations for this proof

$$
F(l):=\|u\|_{\infty, Q_{l}}, G(l):=\|u\|_{\beta, q, Q_{l}}, H(l):=\|u\|_{\alpha, q, Q_{l}} .
$$

Our formal strategy proving the lemma is to apply a Borel-Cantelli type argument. The argument works as following.

(1) We create a sequence of sets $\left\{\mathcal{S}_{i}\right\}$ of the form $\left\{2^{-i} F\left(r_{i}\right)+\sum_{j=1}^{i} c_{j} G\left(r_{j}\right)>a\right\}$ for a sequence of positive numbers $\left\{c_{i}\right\}$ such that $\sum_{i=1}^{\infty} c_{i}<\infty$. Note that $\mathcal{S}_{0}=\left\{F\left(r_{0}\right)>a\right\}$

(2) We will give an estimate of the probability of the event $\mathcal{S}_{0} \cap \mathcal{S}_{m}^{c}$ that is uniform in $m$ by observing $\mathcal{S}_{0} \cap \mathcal{S}_{m}^{c} \subset \bigcup_{i=0}^{m-1}\left(\mathcal{S}_{i} \cap \mathcal{S}_{i+1}^{c}\right)$ and estimating each $\mathbb{P}\left\{\mathcal{S}_{i} \cap \mathcal{S}_{i+1}^{c}\right\}$.

(3) The estimates for $\mathbb{P}\left\{\mathcal{S}_{i} \cap \mathcal{S}_{i+1}^{c}\right\}$ will be provided by first applying LEMMA 5.1 and (4.3) with carefully chosen parameters and then applying (5.3) with appropriate $\epsilon$.

(4) After taking $m \rightarrow \infty$, the uniform estimate we have obtained in (2) will lead to an estimate for the left hand side of (4.4). 
We introduce another auxiliary parameter $\theta>0$ which will also be determined later and pick a sequence of numbers $M_{i}:=M_{0} / \tau^{i \theta}$ for some large $M_{0}>\Gamma(\alpha)$. This sequence will be used as a part of parameters for (4.3) and LEMMA 5.1.

We now choose appropriate values for the sequence $\left\{c_{i}\right\}$. Applying (4.3) with $\Gamma=M_{0}$ on the pair of rectangles $\left(Q_{r_{0}}, Q_{r_{1}}\right)$ and (5.3) with $\epsilon=2^{-1} M_{0}^{-1} V^{-1 / q_{r}} r_{1}^{-n / q}\left(r_{1}-r_{0}\right)^{\lambda}$, we have

$$
\begin{aligned}
& \mathbb{P}\left\{F\left(r_{0}\right)>a, 2^{-1} F\left(r_{1}\right)+\tilde{C}_{\alpha, \beta} M_{0}^{1+\gamma} r^{-(1+\gamma) \lambda} r_{1}^{\gamma n / q} G\left(r_{1}\right) \leq a\right\} \\
\leq & \mathbb{P}\left\{F\left(r_{0}\right)>a, M_{0}\left(r_{1}-r_{0}\right)^{-\lambda} H\left(r_{1}\right) \leq a\right\} \\
\leq & \exp \left\{-M_{0}^{\delta(\alpha)} / r_{1}^{2}\right\} \leq \exp \left\{-M_{0}^{\delta(\alpha)} /\left(4 r^{2}\right)\right\},
\end{aligned}
$$

where $\tilde{C}_{\alpha, \beta}=2^{\gamma} C_{\alpha, \beta} V^{\gamma / q}(1-\tau)^{-(1+\gamma) \lambda}$.

We now have a natural choice of $c_{1}=\tilde{C}_{\alpha, \beta} M_{0}^{1+\gamma} r^{-(1+\gamma) \lambda} r_{1}^{\gamma n / q}$. To find the appropriate value of other $c_{i}$ 's, we look at the third step listed before. In step (3), we want to apply the interpolation inequality (5.3) on $M_{i}\left(r_{i+1}-r_{i}\right)^{-\lambda} H\left(r_{i+1}\right)$ to get

$$
M_{i}\left(r_{i+1}-r_{i}\right)^{-\lambda} H\left(r_{i+1}\right) \leq 2^{-1} F\left(r_{i+1}\right)+2^{i} c_{i} G\left(r_{i+1}\right),
$$

therefore we choose $c_{i}=\tilde{C}_{\alpha, \beta} M_{0}^{1+\gamma} r^{-(1+\gamma) \lambda} r_{i}^{\gamma n / q} \mathcal{K}^{-i+1}$ where $\kappa=2 \tau^{(1+\gamma)(\theta+\lambda)}$.

Now we define the sequence of sets

$$
\mathcal{S}_{i}\left(M_{0}\right):=\left\{2^{-i} F\left(r_{i}\right)+\tilde{C}_{\alpha, \beta} M_{0}^{1+\gamma} r^{-(1+\gamma) \lambda+\gamma n / q} \sum_{j=1}^{i}\left(r_{j} / r\right)^{\gamma n / q} \mathcal{K}^{-j+1} G\left(r_{j}\right)>a\right\},
$$

(5.4) now reads

$$
\mathbb{P}\left\{\mathcal{S}_{0}\left(M_{0}\right) \bigcap \mathcal{S}_{1}^{c}\left(M_{0}\right)\right\} \leq \exp \left\{-M_{0}^{\delta(\alpha)} /\left(4 r^{2}\right)\right\} .
$$

As we have stated before, we have

$$
\mathcal{S}_{0}\left(M_{0}\right) \bigcap \mathcal{S}_{m}^{c}\left(M_{0}\right) \subset \bigcup_{i=0}^{m-1}\left(\mathcal{S}_{i}\left(M_{0}\right) \bigcap \mathcal{S}_{i+1}^{c}\left(M_{0}\right)\right),
$$

thus

$$
\mathbb{P}\left\{\mathcal{S}_{0}\left(M_{0}\right) \bigcap \mathcal{S}_{m}^{c}\left(M_{0}\right)\right\} \leq \sum_{i=0}^{m-1} \mathbb{P}\left\{\mathcal{S}_{i}\left(M_{0}\right) \bigcap \mathcal{S}_{i+1}^{c}\left(M_{0}\right)\right\} .
$$

We estimate the probability $\mathbb{P}\left\{\mathcal{S}_{i}\left(M_{0}\right) \cap \mathcal{S}_{i+1}^{c}\left(M_{0}\right)\right\}$ for $i \geq 1$.

We start by choosing $Z=2^{i} \tilde{C}_{\alpha, \beta} M_{0}^{1+\gamma} r^{-(1+\gamma) \lambda+\gamma n / q} \sum_{j=1}^{i}\left(r_{j} / r\right)^{\gamma n / q} \kappa^{-j+1} G\left(r_{j}\right)$ with $X=F\left(r_{i+1}\right)$ and $Y=H\left(r_{i+1}\right)\left(r_{i+1}-r_{i}\right)^{-\lambda}$ in LEMMA 5.1. We then choose the bounding coefficient $K=$ $2^{-i} \mathcal{K}^{-1} \tilde{C}_{\alpha, \beta}^{-1} M_{0}^{-(1+\gamma)} r^{(1+\gamma) \lambda-\gamma n / q}\left[\sum_{j=1}^{i}\left(r_{j} / r\right)^{\gamma n / q_{\mathcal{K}}}{ }^{-j}\right]^{-1} r_{i+1}^{-2 / \beta+2 / \alpha}\left(r_{i+1}-r_{i}\right)^{-\lambda}$, as Hölder's inequality implies the estimate $H\left(r_{i+1}\right) \geq G\left(r_{i+1}\right) r_{i+1}^{-2 / \beta+2 / \alpha}$. At this moment, all the conditions of LEMMA 5.1 are satisfied with the help of (4.3) if we set $\delta=\delta(\alpha), N_{0}=\Gamma(\alpha)$ and $g=1 /(4 r)^{2}$. We want to use (5.2) with $b=2^{i} a$ and $N=M_{i}$. To do so, we show that $K N^{2} /(K N+1)>N_{0}$ when $M_{0}$ is large. Writing $\tilde{C}=2^{-2 / \beta+2 / \alpha} \tilde{C}_{\alpha, \beta}^{-1}(1-\tau)^{-\lambda}$ and recalling the definition of $\gamma$ and $\lambda$, we have

$$
\begin{aligned}
K N & =M_{0} \tau^{-i \theta} 2^{-i} \mathcal{K}^{-1} \tilde{C}_{\alpha, \beta}^{-1} M_{0}^{-(1+\gamma)} r^{(1+\gamma) \lambda-\gamma n / q}\left[\sum_{j=1}^{i}\left(r_{j} / r\right)^{\gamma n / q} \mathcal{K}^{-j}\right]^{-1} r_{i+1}^{-2 / \beta+2 / \alpha} \tau^{-i \lambda}(1-\tau)^{-\lambda} r^{-\lambda} \\
& =M_{0}^{-\gamma}\left(2 \tau^{\theta+\lambda}\right)^{-i} \mathcal{K}^{-1} \tilde{C}\left[\sum_{j=1}^{i}\left(r_{j} / r\right)^{\gamma n / q} \mathcal{K}^{-j}\right]^{-1}\left(2 r / r_{i+1}\right)^{2 / \beta-2 / \alpha} .
\end{aligned}
$$


From here we first pick $\theta>\frac{2 \gamma}{1-\gamma} \lambda$ so that $\frac{3+\gamma}{2} \theta+\lambda>(\theta+\lambda)(\gamma+1)$, then pick $\tau<1$ such that $2 \tau^{\frac{3+\gamma}{2}} \theta+\lambda=1$. The choices of $\theta$ and $\tau$ also guarantee $\kappa>1$. Writing $C=2^{-\gamma n / q}\left(1-\kappa^{-1}\right) \tilde{C}$, we estimate $K N$ as following,

$$
K N \geq M^{-\gamma}\left(2 \tau^{\theta+\lambda}\right)^{-i} C\left(\sum_{j=0}^{i-1} \kappa^{-j}\right)^{-1}\left(1-\kappa^{-1}\right)^{-1} \geq M^{-\gamma}\left(2 \tau^{\theta+\lambda}\right)^{-i} C
$$

Therefore we have, when $M_{0}$ is large,

$$
\begin{aligned}
K N^{2} /(K N+1) & =N(K N) /(K N+1) \geq M_{0} \tau^{-i \theta} C\left[C+\left(2 \tau^{\theta+\lambda}\right)^{i} M_{0}^{\gamma}\right]^{-1} \\
& =M_{0}^{1-\gamma} \tau^{-i(1-\gamma) \theta / 2}\left[M_{0}^{-\gamma} \tau^{i(1+\gamma) \theta / 2}+C^{-1}\left(2 \tau^{(3+\gamma) \theta / 2+\gamma}\right)^{i}\right]^{-1} \\
& \geq M_{0}^{1-\gamma} \tau^{-i(1-\gamma) \theta / 2}\left(1+C^{-1}\right)^{-1} \\
& >N_{0} .
\end{aligned}
$$

From here, by (5.2) we obtain,

$$
\begin{aligned}
& \mathbb{P}\left\{F\left(r_{i}\right)+2^{i} \tilde{C}_{\alpha, \beta} M_{0}^{1+\gamma} r^{-(1+\gamma) \lambda+\gamma n / q} \sum_{j=1}^{i}\left(r_{j} / r\right)^{\gamma n / q} \mathcal{K}^{-j+1} G\left(r_{j}\right)>2^{i} a\right. \\
& \left.M_{0} \tau^{-i \theta}\left(r_{i}+1-r_{i}\right)^{-\lambda} H\left(r_{i+1}\right)+2^{i} \tilde{C}_{\alpha, \beta} M_{0}^{1+\gamma} r^{-(1+\gamma) \lambda+\gamma n / q} \sum_{j=1}^{i}\left(r_{j} / r\right)^{\gamma n / q} \mathcal{K}^{-j+1} G\left(r_{j}\right) \leq 2^{i} a\right\} \\
& \leq \exp \left\{-\left(K N^{2} /(K N+1)\right)^{\delta(\alpha)} /\left(4 r^{2}\right)\right\} \\
& \leq \exp \left\{-\left(M_{0}^{1-\gamma} \tau^{-i(1-\gamma) \theta / 2}\left(1+C^{-1}\right)^{-1}\right)^{\delta(\alpha)} /\left(4 r^{2}\right)\right\} \\
& \leq \exp \left\{-\left(M_{0} \tau^{-i \theta}\right)^{\delta(\alpha)(1-\gamma) / 2} /\left(4 r^{2}\right)\right\} .
\end{aligned}
$$

It is worth noting that the estimates so far are uniform for all $i$ and $r \in(0,1 / 2)$ when $M_{0}$ is large.

We now use (5.3) with $\epsilon=2^{-1} M_{0}^{-1} V^{-1 / q} r_{i+1}^{-n / q}\left(r_{i+1}-r_{i}\right)^{\lambda}$ and $l=r_{i+1}$. The last estimate gives,

$$
\mathbb{P}\left\{\mathcal{S}_{i}\left(M_{0}\right) \bigcap \mathcal{S}_{i+1}^{c}\left(M_{0}\right)\right\} \leq \exp \left\{-\left(M_{0} \tau^{-i \theta}\right)^{\delta(\alpha)(1-\gamma) / 2} /\left(4 r^{2}\right)\right\}
$$

which further leads to

$$
\mathbb{P}\left\{\mathcal{S}_{0}\left(M_{0}\right) \bigcap \mathcal{S}_{m}^{c}\left(M_{0}\right)\right\} \leq \exp \left\{-M_{0}^{\delta(\alpha)} /\left(4 r^{2}\right)\right\}+\sum_{i=1}^{m-1} \exp \left\{-\left(M_{0} \tau^{-i \theta}\right)^{\delta(\alpha)(1-\gamma) / 2} /\left(4 r^{2}\right)\right\}
$$

Recalling that $\gamma=-1+\alpha / \beta$ and $\lambda=2 / \alpha+n / q$, we have the identity $(1+\gamma) \lambda-\gamma n / q=$ $2 / \beta+n / q$. This means the last inequality we have actually says,

$$
\begin{aligned}
\mathbb{P}\{F(r)>a, & \left.2^{-m} F\left(r_{m}\right)+\tilde{C}_{\alpha, \beta} M_{0}^{1+\gamma} r^{-(n / q+2 / \beta)} \sum_{j=0}^{m-1}\left(r_{j} / r\right)^{\gamma n / q} \kappa^{-j} G\left(r_{j+1}\right) \leq a\right\} \\
& \leq \exp \left\{-M_{0}^{\delta(\alpha)} /\left(4 r^{2}\right)\right\}+\sum_{i=1}^{m-1} \exp \left\{-\left(M_{0} \tau^{-i \theta}\right)^{\delta(\alpha)(1-\gamma) / 2} /\left(4 r^{2}\right)\right\},
\end{aligned}
$$


which implies

$$
\begin{aligned}
\mathbb{P}\{F(r)>a, & \left.2^{-m} F(2 r)+2^{\gamma n / q} \tilde{C}_{\alpha, \beta}(1-\kappa) M_{0}^{1+\gamma} r^{-(n / q+2 / \beta)} G(2 r)<a\right\} \\
& \leq \exp \left\{-M_{0}^{\delta(\alpha)} /\left(4 r^{2}\right)\right\}+\sum_{i=1}^{m-1} \exp \left\{-\left(M_{0} \tau^{-i \theta}\right)^{\delta(\alpha)(1-\gamma) / 2} /\left(4 r^{2}\right)\right\} \\
& \leq \exp \left\{-M_{0}^{\delta^{\prime}} /\left(4 r^{2}\right)\right\}
\end{aligned}
$$

when $M_{0}$ is sufficiently large for $\delta^{\prime}=(1-\gamma) \delta(\alpha) / 4$.

Since $u \in L^{\alpha}\left([0,1], L^{q}\left(B_{1}\right)\right)$ almost surely and $2 r<1$, by choosing sufficiently large $a$ and $\Gamma$ in (4.3), we obtain

$$
\mathbb{P}\left\{\|u\|_{\infty, Q_{2 r}}<\infty\right\}=1 .
$$

Taking $m \rightarrow \infty$, Fatou's Lemma and (5.6) gives,

$$
\mathbb{P}\left\{F(r)>a, 2^{\gamma n / q} \tilde{C}_{\alpha, \beta}(1-\kappa) M_{0}^{1+\gamma} r^{-(n / q+2 / \beta)} G(2 r)<a\right\} \leq \exp \left\{-M_{0}^{\delta^{\prime}} /\left(4 r^{2}\right)\right\} .
$$

This implies

$$
\mathbb{P}\left\{F(r)>a, 2^{1+\gamma n / q} \tilde{C}_{\alpha, \beta}(1-\kappa) M_{0}^{1+\gamma} r^{-(n / q+2 / \beta)} G(2 r) \leq a\right\} \leq \exp \left\{-M_{0}^{\delta^{\prime}} /\left(4 r^{2}\right)\right\} .
$$

The inequality (5.7) implies (4.4) for $\delta(\beta)=\delta^{\prime} /(2(1+\gamma))$ and sufficiently large $\Gamma(\beta)$ for the case $0<r<1 / 2$.

It remains to deal with the case $r=1 / 2$. (5.7) with $r=1 / 2-\epsilon$ implies

$$
\mathbb{P}\left\{F(1 / 2-\epsilon)>a, 2^{1+\gamma n / q} \tilde{C}_{\alpha, \beta}(1-\kappa) M_{0}^{1+\gamma}(1 / 2-\epsilon)^{-(n / q+2 / \beta)} G(1)<a\right\} \leq \exp \left\{-M_{0}^{\delta^{\prime}}\right\} .
$$

Letting $\epsilon=1 / k$ and $k \rightarrow \infty$, we get from Fatou's lemma,

$$
\mathbb{P}\left\{F(1 / 2)>a, 2^{1+\gamma n / q} \tilde{C}_{\alpha, \beta}(1-\kappa) M_{0}^{1+\gamma}(1 / 2)^{-(n / q+2 / \beta)} G(1)<a\right\} \leq \exp \left\{-M_{0}^{\delta^{\prime}}\right\} .
$$

The last inequality implies

$$
\mathbb{P}\left\{F(1 / 2)>a, 2^{2+\gamma n / q} \tilde{C}_{\alpha, \beta}(1-\kappa) M_{0}^{1+\gamma}(1 / 2)^{-(n / q+2 / \beta)} G(1) \leq a\right\} \leq \exp \left\{-M_{0}^{\delta^{\prime}}\right\} .
$$

This finishes the proof for LEMMA 4.4.

For LEMMA 4.5, we need to use a different interpolation inequality

$$
\begin{aligned}
\|u\|_{p, \alpha, Q_{l}} & \leq\|u\|_{p, \infty, Q_{l}}^{1-\beta / \alpha}\|u\|_{p, \beta, Q_{l}}^{\beta / \alpha} \\
& \leq \epsilon\|u\|_{p, \infty, Q_{l}}+C_{\alpha, \beta} \epsilon^{-\gamma}\|u\|_{p, \beta, Q_{l}} \leq \epsilon l^{2 / p}\|u\|_{\infty, Q_{l}}+C_{\alpha, \beta} \epsilon^{-\gamma}\|u\|_{p, \beta, Q_{l}} .
\end{aligned}
$$

The Borel-Cantalli argument will be applied to the following sequence of sets

$$
\overline{\mathcal{S}}_{i}\left(M_{0}\right):=\left\{2^{-i}\|u\|_{\infty, Q_{r_{i}}}+\bar{C}_{\alpha, \beta} M_{0}^{1+\gamma} r^{-(1+\gamma) \bar{\lambda}+2 \gamma / p} \sum_{j=0}^{i-1}\left(r_{j} / r\right)^{2 \gamma / p_{\mathcal{K}^{-j}}}\|u\|_{p, \beta, Q_{r_{j+1}}}>a\right\}
$$

with $\bar{C}_{\alpha, \beta}=2^{\gamma} C_{\alpha, \beta}(1-\tau)^{-(1+\gamma) \bar{\lambda}}$ and $\bar{\lambda}=2 / p+n / \alpha$.

The rest of the proof is identical to the one for LEMMA 4.4 . 


\section{REVERSE CAUCHY-SCHWARZ TYPE INEQUALITY}

In this section, we will prove PROPOSITION 2.5, the reverse Cauchy-Schwartz type inequality. Recalling the following definition for all positive function $v>0$ and space-time regions $D_{1}$ and $D_{2}$,

$$
\mathcal{F}[v, \alpha]_{D_{1}, D_{2}}:=\left(\int_{D_{1}} v^{-\alpha} d x d t\right)\left(\int_{D_{2}} v^{\alpha} d x d t\right),
$$

we restate our goal here.

Proposition 6.1. Let $u$ be a non-negative super-solution of (1.1) in $[0,2] \times B_{1}$. Given $t \in(0,1)$, for every $\epsilon>0$, there exist constants $\alpha_{\epsilon}$ and $K_{\epsilon}$ depending only on $n, l, \Lambda, t$ and $\epsilon$ such that $\forall \mu>0$

$$
\mathbb{P}\left\{\mathcal{F}\left[u+\mu, \alpha_{\epsilon}\right]_{D_{0}^{+}, D_{0}^{-}}^{1 / \alpha_{\epsilon}}>K_{\epsilon}\right\}<\epsilon .
$$

Here $D_{0}^{+}=\left(2-t^{2}, 2\right) \times B_{t}$ and $D_{0}^{-}=\left(0, t^{2}\right) \times B_{t}$.

To better present our idea, we assume $t=1 / 2$ for now. Our proof will work for every $t \in(0,1)$ with minor adjustments and we will point out the differences after the proof.

We write $h^{\mu}=-\log (u+\mu)$ and we will prove a tail estimate for $\int_{D^{+}} e^{v h^{\mu}} d x d t \int_{D^{-}} e^{-v h^{\mu}} d x d t$ regardless of $\mu$ when $v$ is small. The proof of such estimate relies heavily on a variant of the parabolic John-Nirenberg inequality. To present the variant, we define a few collections of spacetime rectangular regions as following.

We first create a large collection of cubes within $[0,2] \times B_{1}$ starting from $C_{0}=(0,2) \times B_{1 / 2}$. We start by defining $\mathscr{C}_{0}=\left\{C_{0}\right\}$. For every cube $C$ assuming the form $(l-4 s, l+4 s) \times B_{z}(w)$, we write $C^{+}:=(l, l+4 s) \times B_{z}(w), C^{-}:=(l-4 s, l) \times B_{z}(w), D^{+}:=(l+3 s, l+4 s) \times B_{z}(w), D^{-}:=$ $(l-4 s, l-3 s) \times B_{z}(w), I^{+}:=(l+2 s, l+4 s) \times B_{z}(w)$ and $I^{-}:=(l-4 s, l-2 s) \times B_{z}(w)$ as in Fig 7 In this way, we have $C_{0}^{+}, C_{0}^{-}, D_{0}^{+}, D_{0}^{-}, I_{0}^{+}$and $I_{0}^{-}$defined, and we define $\mathscr{C}_{0}^{+}, \mathscr{C}_{0}^{-}, \mathscr{D}_{0}^{+}, \mathscr{D}_{0}^{-}, \mathscr{I}_{0}^{+}$and $\mathscr{I}_{0}^{-}$as the collection made up of each of them, respectively.
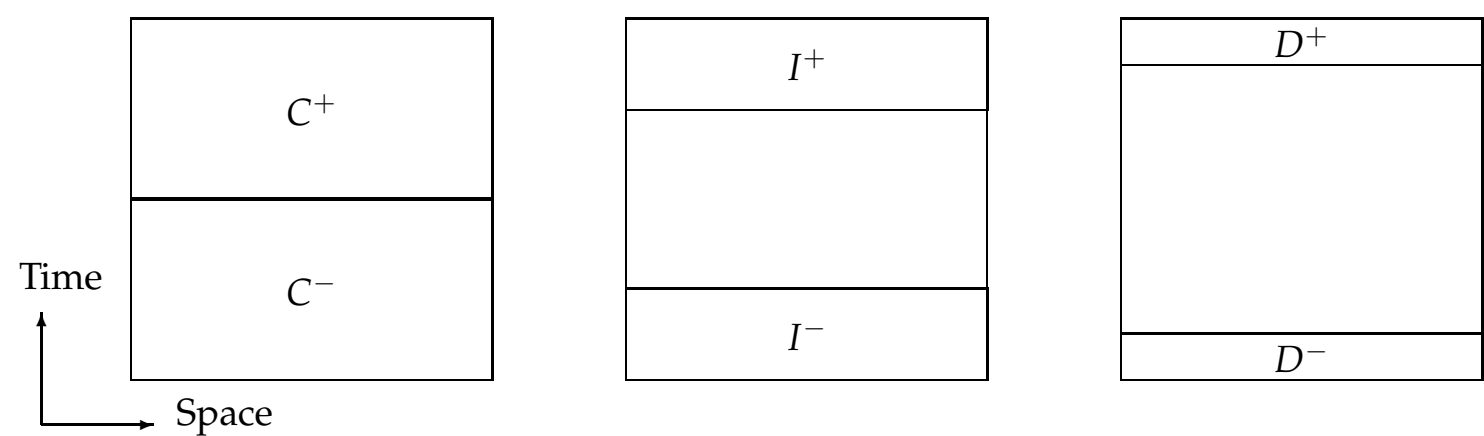

FIGURE 7. The whole cube is $C, C^{ \pm}$occupy the upper/lower half, $D^{ \pm}$occupy the upper/lower one eighth, and $I^{ \pm}$occupy the upper/lower quarter.

Now assume $\mathscr{C}_{i}$ has been defined for some $i$, we divide each cube in $\mathscr{D}_{i}^{+}$and $\mathscr{D}_{i}^{-}$into $4^{n+2}$ congruent pieces by dividing the time interval it spans into 16 equal pieces and space interval in each dimension into 4 equal pieces. For any one of these smaller cubes, if it comes from dividing some $D_{i}^{+}(k)$ in $\mathscr{D}_{i}^{+}$, we think of it as the $D^{+}$of some $C$ and put that $C$ into $\mathscr{C}_{i+1}$; otherwise we think of it as the $D^{-}$of some $C$ and put that $C$ into $\mathscr{C}_{i+1}$. After this is done for all the smaller cubes coming from the division, we define $\mathscr{C}_{i+1}^{+}, \mathscr{C}_{i+1}^{-}, \mathscr{D}_{i+1}^{+}, \mathscr{D}_{i+1}^{-}, \mathscr{I}_{i+1}^{+}$and $\mathscr{I}_{i+1}^{-}$as the collection of the 
respective cubes corresponding to the ones in $\mathscr{C}_{i+1}$. We repeat this process and define $\mathscr{C}\left(C_{0}\right)=$ $\bigcup_{j=1}^{\infty} \mathscr{C}_{j}$.

We now define a new collection $\mathscr{C}^{\prime}\left(C_{0}\right)$. The way to define it is almost identical to the process creating $\mathscr{C}\left(C_{0}\right)$. Again we start with $C_{0}$, but this time we proceed by dividing $I^{ \pm}$into $4^{n+2}$ congruent pieces instead of $D^{ \pm}$. After constructing $\mathscr{C}^{\prime}\left(C_{0}\right)$, we note that such construction can actually be done starting with any cube in $\mathscr{C}\left(C_{0}\right)$, and we write $\mathscr{C}^{\prime}:=\bigcup_{C_{j}(i) \in \mathscr{C}\left(C_{0}\right)} \mathscr{C}^{\prime}\left(C_{j}(i)\right)$. For the convenience of later calculation, we re-arrange the labels in $\mathscr{C}^{\prime}$ so any $C_{m}(k)$ from it has spatial radius $2^{-2 m-1}$.

Our parabolic John-Nirenberg inequality takes the following form.

Proposition 6.2. Assume $f$ is an $L^{2}$ function on $(0,2) \times B_{1}$. Suppose that we have a constant $A>0$ such that there exists $a_{C_{j}(i)}$ for every cube $C_{j}(i) \in \mathscr{C}^{\prime}$ satisfying the following inequalities on the corresponding cubes $C_{j}^{ \pm}(i)$,

$$
\begin{aligned}
& \frac{1}{\left|C_{j}^{+}(i)\right|} \int_{C_{j}^{+}(i)} \sqrt{\left(f(t, x)-a_{C_{j}(i)}\right)^{+}} d x d t \leq A, \\
& \frac{1}{\left|C_{j}^{-}(i)\right|} \int_{C_{j}^{-}(i)} \sqrt{\left(a_{C_{j}(i)}-f(t, x)\right)^{+}} d x d t \leq A .
\end{aligned}
$$

Then there exist two positive constants $B$ and $b$ depending only on the dimension $n$ and $A$ such that for every $\alpha>0$,

$$
\begin{aligned}
& \left|\left\{(t, x) \in D_{0}^{+} \mid\left(f(t, x)-a_{C_{0}}\right)^{+}>\alpha\right\}\right| \leq B e^{-\frac{b \alpha}{A}}\left|D_{0}^{+}\right|, \\
& \left|\left\{(t, x) \in D_{0}^{-} \mid\left(a_{C_{0}}-f(t, x)\right)^{+}>\alpha\right\}\right| \leq B e^{-\frac{b x}{A}}\left|D_{0}^{-}\right| .
\end{aligned}
$$

Furthermore, for any $0<v<\frac{b}{A}$,

$$
\int_{D_{0}^{+}} e^{v f} d x d t \int_{D_{0}^{-}} e^{-v f} d x d t \leq B^{2} v^{2}\left|D_{0}^{+}\right|\left|D_{0}^{-}\right|\left(\int_{0}^{\infty} e^{\left(v-\frac{b}{A}\right) \alpha} d \alpha\right)^{2} .
$$

Remark 6.3. We note here that $\mathscr{D}_{j}^{+}$is made up of $2^{j} \times 4^{j(n+2)}$ elements with spatial radius $2^{-1} \times 4^{-j}$. As for the collection $\mathscr{C}^{\prime}$, we note that any cube in $\mathscr{C}^{\prime}$ with spatial radius $2^{-1} \times 4^{-j}$ either comes from dividing cubes with spatial radius $2^{-1} \times 4^{-j+1}$ into $4^{n+2}$ pieces or lives in $\mathscr{C}\left(C_{0}\right)$. We denote by $x_{j}$ the number of cubes with spatial radius $2^{-1} \times 4^{-j}$ in $\mathscr{C}^{\prime}$, the previous observation gives $x_{j}=2 \times 4^{n+2} x_{j-1}+2^{j} \times 4^{(n+2) j}$ which leads to $x_{j} \leq 4^{(n+3) j}$.

The proof of this parabolic John-Nirenberg inequality is identical to the classical ones as in Fabes and Garofalo [3] and Moser [7]. We therefore only sketch the proof and omit the details here.

The main tools in proving PROPOSITION 6.2 are the following two lemmas.

Lemma 6.4. Assume $f$ is an $L^{2}$ function on $(0,2) \times B_{1}$. Suppose we have a constant $A>0$ such that there exists $a_{C_{j}(i)}$ for every cube $C_{j}(i) \in \mathscr{C}$ satisfying the following two inequalities on the corresponding cubes $I_{j}^{ \pm}(i)$,

$$
\begin{aligned}
& \frac{1}{\left|I_{j}^{+}(i)\right|} \int_{I_{j}^{+}(i)}\left(f(t, x)-a_{C_{j}(i)}\right)^{+} d x d t \leq A, \\
& \frac{1}{\left|I_{j}^{-}(i)\right|} \int_{I_{j}^{-}(i)}\left(a_{C_{j}(i)}-f(t, x)\right)^{+} d x d t \leq A .
\end{aligned}
$$


Then there exist two positive dimensional constants $B$ and $b$ such that for every $\alpha>0$,

$$
\begin{aligned}
& \left|\left\{(t, x) \in D_{0}^{+} \mid\left(f(t, x)-a_{C_{0}}\right)^{+}>\alpha\right\}\right| \leq B e^{-\frac{b x}{A}}\left|D_{0}^{+}\right|, \\
& \left|\left\{(t, x) \in D_{0}^{-} \mid\left(a_{C_{0}}-f(t, x)\right)^{+}>\alpha\right\}\right| \leq B e^{-\frac{b x}{A}}\left|D_{0}^{-}\right| .
\end{aligned}
$$

Lemma 6.5. Assume $f$ is an $L^{2}$ function on $(0,2) \times B_{1}$. Suppose that we have a constant $A^{\prime}>0$ such that there exists $a_{C_{j}(i)}$ for every cube $C_{j}(i) \in \mathscr{C}^{\prime}$ satisfying the following inequalities on the corresponding cubes $C_{j}^{ \pm}(i)$,

$$
\begin{aligned}
& \frac{1}{\left|C_{j}^{+}(i)\right|} \int_{C_{j}^{+}(i)} \sqrt{\left(f(t, x)-a_{C_{j}(i)}\right)} d x d t \leq A^{\prime}, \\
& \frac{1}{\left|C_{j}^{-}(i)\right|} \int_{C_{j}^{-}(i)} \sqrt{\left(a_{C_{j}(i)}-f(t, x)\right)+} d x d t \leq A^{\prime} .
\end{aligned}
$$

Then there exists two positive dimensional constants $B^{\prime}$ and $b^{\prime}$ such that for every $\alpha>0$ and $C_{m}(k) \in$ $\mathscr{C}\left(C_{0}\right)$, the following two inequalities are satisfied on the corresponding cubes $I_{m}^{ \pm}(k)$,

$$
\begin{aligned}
& \left|\left\{(t, x) \in I_{m}^{+}(k) \mid\left(f(t, x)-a_{C_{m}(k)}\right)^{+}>\alpha\right\}\right| \leq B^{\prime} e^{-b^{\prime}\left(\frac{\alpha}{A^{\prime}}\right)^{\frac{1}{2}}}\left|I_{m}^{+}(k)\right|, \\
& \left|\left\{(t, x) \in I_{m}^{-}(k) \mid\left(a_{C_{m}(k)}-f(t, x)\right)^{+}>\alpha\right\}\right| \leq B^{\prime} e^{-b^{\prime}\left(\frac{\alpha}{A^{\prime}}\right)^{\frac{1}{2}}}\left|I_{m}^{-}(k)\right| .
\end{aligned}
$$

These two lemmas are the exact copies of [3, Theorem 1 and 2] with the space-time rectangular regions used in the proofs specified. Therefore their proofs will not be included in our article. With these two lemmas, we can provide a short proof of PROPOSITION 6.2

Proof of Proposition 6.2 From LemMa 6.5, we have on each $C_{m}(k) \in \mathscr{C}_{0}$,

$$
\int_{I_{m}^{+}(k)}\left(f(t, x)-a_{C_{m}(k)}\right)^{+} d x d t \leq\left|I_{m}^{+}(k)\right| \int_{0}^{\infty} B^{\prime} e^{-b^{\prime}\left(\frac{\alpha}{A^{\prime}}\right)^{\frac{1}{2}}} d \alpha,
$$

hence (6.4) is satisfied. A similar argument shows that the other inequality in the assumptions of LEMMA 6.4 is also satisfied with $A=\int_{0}^{\infty} B^{\prime} e^{-b^{\prime}\left(\frac{\alpha}{A^{\prime}}\right)^{\frac{1}{2}}} d \alpha$.

At this moment, LEMMA 6.4 can be applied. We have for each $v<\frac{b}{A}$,

$$
\begin{gathered}
\int_{D_{0}^{+}} e^{v f} d x d t \leq \int_{D_{0}^{+}} e^{v a_{C_{0}}} e^{v\left(f-a_{C_{0}}\right)^{+}} d x d t \leq B e^{v a C_{0}} v\left|D_{0}^{+}\right| \int_{0}^{\infty} e^{\left(v-\frac{b}{A}\right) \alpha} d \alpha \\
\int_{D_{0}^{-}} e^{-v f} d x d t \leq \int_{D_{0}^{-}} e^{-v a_{C_{0}}} e^{v\left(a_{C_{0}}-f\right)^{+}} d x d t \leq B e^{-v a_{C_{0}}} v\left|D_{0}^{-}\right| \int_{0}^{\infty} e^{\left(v-\frac{b}{A}\right) \alpha} d \alpha .
\end{gathered}
$$

Therefore the proposition is proved.

With Proposition 6.2 in hand, our goal now is to find suitable $a_{C_{j}(i)}$ s and $A$ satisfying the assumptions in the proposition for $f=h^{\mu}$. Since we have a stochastic perturbation term in (1.1), we cannot expect an almost sure result with fixed $A$ and deterministic $a_{C_{j}(i)}$ s. However, we can get an almost sure statement including a random perturbation, and then bound the perturbation on a large probability.

To state our results, a few extra notations need to be introduced. We write $r_{j}=2^{-1} \times 4^{-j}$ for simplicity and pick a smooth cut-off function $\phi$ which is 1 on $B_{1 / 2}$, is 0 outside $B_{3 / 4}$ with convex 
level set, and is bounded between $[0,1]$. For any $C_{j}(i) \in \mathscr{C}^{\prime}$, its spatial radius is $r_{j}$ and there exists $(s, x)$ such that

$$
C_{j}(i)=\left(s-4 r_{j}^{2}, s+4 r_{j}^{2}\right) \times B_{r_{j}}(x) .
$$

On $C_{j}(i)$, denoting by $\phi_{B_{r_{j}}(x)}(y):=\phi\left(\left(2 r_{j}\right)^{-1}(y-x)\right)$ the cut-off function scaled to $B_{r_{j}}(x)$, recalling $h^{\mu}=-\log (u+\mu)$ and introducing $\left|V\left(C_{j}(i), \phi\right)\right|=\int_{B_{3 r_{j} / 2}(x)} \phi_{B_{r_{j}}(x)}^{2}(y) d y$, we define

$$
\left\{\begin{array}{l}
M_{C_{j}(i)}^{\mu}(t):=\sum_{i} \int_{s}^{s+t} \frac{1}{\left|V\left(C_{j}(i), \phi\right)\right|} \int_{B_{3 r_{j} / 2}(x)} \tilde{g}_{i}^{\mu}(\tau, y, u ; \omega) \phi_{B_{r_{j}}(x)}^{2}(y) d y d w_{\tau}^{i}, \\
H_{C_{j}(i)}^{\mu}(t):=\frac{1}{\left|V\left(C_{j}(i), \phi\right)\right|} \int_{B_{3 r_{j} / 2}(x)} h^{\mu}(t+s, y ; \omega) \phi_{B_{r_{j}}(x)}^{2}(y) d y,
\end{array}\right.
$$

where $\tilde{g}_{i}^{\mu}(t, x, u ; \omega):=g_{i}(t, x, u ; \omega)(u+\mu)^{-1}$.

Remark 6.6. The quadratic variation process $\left\langle M_{C_{j}(i)}^{\mu}\right\rangle_{t}$ is bounded by constant times of $t$.

We have the following almost sure result.

Lemma 6.7. Let $u$ be a non-negative super-solution to (1.1) in $[0,2] \times B_{1}$. There exists a constant $\bar{A}$ depending only $n, l, \Lambda$ such that for every $C_{j}(i) \in \mathscr{C}^{\prime}$, we can find a random variable $a_{C_{j}(i)}$ satisfying

$$
\frac{1}{\left|C_{j}^{+}(i)\right|} \int_{C_{j}^{+}(i)} \sqrt{\left(h^{\mu}-M_{C_{j}(i)}^{\mu}-a_{C_{j}(i)}\right)^{+}} d x d t \leq \bar{A} \quad \text { a.s. }
$$

and

$$
\frac{1}{\left|C_{j}^{-}(i)\right|} \int_{C_{j}^{-}(i)} \sqrt{\left(M_{C_{j}(i)}^{\mu}+a_{C_{j}(i)}-h^{\mu}\right)^{+}} d x d t \leq \bar{A} \quad \text { a.s.. }
$$

Proof. We will use $(\cdot, \cdot)$ to denote the inner product on $\mathbb{R}^{n}$. By direct calculation, $h^{\mu}$ is a subsolution of

$$
d h^{\mu}=\operatorname{div}\left(\mathbb{A} \nabla h^{\mu}\right) d t-\left(\mathbb{A} \nabla h^{\mu}, \nabla h^{\mu}\right) d t+\tilde{f}^{\mu} d t+\tilde{g}_{i}^{\mu} d w_{t}^{i}
$$

with

$$
\tilde{f}^{\mu}(t, x, u ; \omega)=f(t, x, u ; \omega)(u+\mu)^{-1}+2^{-1}\left|\tilde{g}^{\mu}\right|_{\ell^{2}}^{2} .
$$

Fixing any $C_{j}(i) \in \mathscr{C}^{\prime}$, by testing (6.11) with $\phi_{B_{r_{j}}(x)}^{2}(y)$, we have for any $t_{1}$ and $t_{2}$ such that $s-4 r_{j}^{2}<t_{1} \leq t_{2}<s+4 r_{j}^{2}$,

$$
\begin{aligned}
& \int_{B_{1}} h^{\mu}\left(t_{2}\right) \phi_{B_{r_{j}}(x)}^{2} d y-\int_{B_{1}} h^{\mu}\left(t_{1}\right) \phi_{B_{r_{j}}(x)}^{2} d y+\int_{t_{1}}^{t_{2}} \int_{B_{1}}\left(\mathbb{A} \nabla h^{\mu}, \nabla h^{\mu}\right) \phi_{B_{r_{j}}(x)}^{2} d y d \tau \\
& \leq-\int_{t_{1}}^{t_{2}}\left\langle\mathbb{A} \nabla h^{\mu}, \nabla\left(\phi_{B_{r_{j}}(x)}^{2}\right)\right\rangle d \tau+\int_{t_{1}}^{t_{2}} \int_{B_{1}} \tilde{f}^{\mu} \phi_{B_{r_{j}}(x)}^{2} d y d \tau+\sum_{i} \int_{t_{1}}^{t_{2}} \int_{B_{1}} \tilde{g}_{k}^{\mu} \phi_{B_{r_{j}}(x)}^{2} d y d w_{\tau}^{k} .
\end{aligned}
$$

Applying the Cauchy-Schwarz inequality to the first term on the right hand side, the above inequality implies

$$
\begin{aligned}
\int_{B_{3 r_{j} / 2}(x)} h^{\mu}\left(t_{2}\right) \phi_{B_{r_{j}}(x)}^{2} d y & -\int_{B_{3 r_{j} / 2}(x)} h^{\mu}\left(t_{1}\right) \phi_{B_{r_{j}}(x)}^{2} d y+\frac{1}{2} \int_{t_{1}}^{t_{2}} \int_{B_{3 r_{j} / 2}(x)}\left(\mathbb{A} \nabla h^{\mu}, \nabla h^{\mu}\right) \phi_{B_{r_{j}}(x)}^{2} d y d \tau \\
\leq & 2 \int_{t_{1}}^{t_{2}}\left\langle\nabla \phi_{B_{r_{j}}(x)}, \mathbb{A} \nabla \phi_{B_{r_{j}}(x)}\right\rangle d y d \tau \\
& +\int_{t_{1}}^{t_{2}} \int_{B_{3 r_{j} / 2}(x)} \tilde{f}^{\mu} \phi_{B_{r_{j}}(x)}^{2} d y d \tau+\sum_{i} \int_{t_{1}}^{t_{2}} \int_{B_{3 r_{j} / 2}(x)} \tilde{g}_{k}^{\mu} \phi_{B_{r_{j}}(x)}^{2} d y d w_{\tau}^{k} .
\end{aligned}
$$


Using the uniform ellipticity of $\mathbb{A}$ and the growth bound of $f$, we obtain for some positive constants $C_{1}, C_{2}$ and $C_{3}$,

$$
\begin{aligned}
\int_{B_{3 r_{j} / 2}(x)} h^{\mu}\left(t_{2}\right) \phi_{B_{r_{j}}(x)}^{2} d y- & \int_{B_{3 r_{j} / 2}(x)} h^{\mu}\left(t_{1}\right) \phi_{B_{r_{j}}(x)}^{2} d y+C_{1}^{-1} \int_{t_{1}}^{t_{2}}\left\|\phi_{B_{r_{j}}(x)} \nabla h^{\mu}\right\|_{2, B_{3 r_{j} / 2}(x)}^{2} d s \\
& \leq\left(C_{2} r_{j}^{-2}+C_{3}\right)\left|V\left(C_{j}(i), \phi\right)\right|\left(t_{2}-t_{1}\right)+\sum_{i} \int_{t_{1}}^{t_{2}} \int_{B_{3 r_{j} / 2}(x)} \tilde{g}_{k}^{\mu} \phi_{B_{r_{j}}(x)}^{2} d y d w_{\tau}^{k} .
\end{aligned}
$$

Dividing the above inequality by $\left|V\left(C_{j}(i), \phi\right)\right|$ and applying a weighted Poincarés inequality (see [7. Lemma 3, Page 120]) to the third term on the left hand side, we have the following differential inequality for a different $C_{1}$,

$$
d H_{C_{j}(i)}^{\mu}+\left(\frac{C_{1}^{-1} r_{j}^{-2}}{\left|V\left(C_{j}(i), \phi\right)\right|} \int_{B_{3 r_{j} / 2}(x)}\left(h^{\mu}-H_{C_{j}(i)}^{\mu}\right)^{2} \phi_{B_{r_{j}}(x)}^{2} d y\right) d t \leq\left(C_{2} r_{j}^{-2}+C_{3}\right) d t+d M_{C_{j}(i)}^{\mu}
$$

We emphasize that the above inequality should be interpreted in the integral form, and this rule applies to all the differential inequalities below.

We define the following two stochastic processes

$$
z(t, y):=h^{\mu}(s+t, y)-M_{C_{j}(i)}^{\mu}(t)-\left(C_{2} r_{j}^{-2}+C_{3}\right) t-H_{C_{j}(i)}^{\mu}(0)
$$

and

$$
Z(t):=H_{C_{j}(i)}^{\mu}(t)-H_{C_{j}(i)}^{\mu}(0)-M_{C_{j}(i)}^{\mu}(t)-\left(C_{2} r_{j}^{-2}+C_{3}\right) t .
$$

The inequality (6.12) is equivalent to

$$
d Z+\frac{C_{1}^{-1} r_{j}^{-2}}{\left|V\left(C_{j}(i), \phi\right)\right|} \int_{B_{3 r_{j} / 2(x)}}(z-Z)^{2} \phi_{B_{r_{j}}(x)}^{2} d y d t \leq 0, \quad Z(0)=0 .
$$

We now extract a growth bound for the level set of $z$ from (6.13). The inequality implies immediately for all non-negative $t$,

$$
Z(t) \leq 0, \quad \mathbb{P} \text { almost surely. }
$$

For arbitrary $a \geq 1$, we write $E_{a}(t):=\left\{y \in B_{r_{j}}(x): z(t, y)>a\right\}$. On $E_{a}(t)$, we have $0<(a-$ $Z) \leq(z-Z)$, thus $(a-Z)^{2}\left|E_{a}(t)\right| \leq \int_{B_{3 r_{j} / 2}(x)}(z-Z)^{2} \phi_{B_{r_{j}}(x)}^{2} d y$. Then it follows from (6.13) that

$$
\frac{1}{(a-Z)^{2}} d Z+\frac{C_{1}^{-1} r_{j}^{-2}}{\left|V\left(C_{j}(i), \phi\right)\right|}\left|E_{a}(t)\right| d t \leq 0 .
$$

By Itô's formula, $d \frac{1}{a-Z}=\frac{1}{(a-Z)^{2}} d Z+\frac{1}{(a-Z)^{3}} d\left\langle M_{C_{j}(i)}^{\mu}\right\rangle_{t}$, so we have

$$
\frac{C_{1}^{-1} r_{j}^{-2}}{\left|V\left(C_{j}(i), \phi\right)\right|}\left|E_{a}(t)\right| d t \leq-d \frac{1}{a-Z}+\frac{1}{(a-Z)^{3}} d\left\langle M_{C_{j}(i)}^{\mu}\right\rangle_{t} .
$$

Using the above inequality in its integral form between 0 and $4 r_{j}^{2}$ and applying the quadratic variation bound on $M^{\mu}$, we obtain with the fact $r_{j} \leq 1 / 2$,

$$
\left|\left\{(t, y) \in C_{j}^{+}(i): z(t-s, y)>a\right\}\right| \leq \frac{C_{4}}{a}\left|C_{j}^{+}(i)\right|
$$

for a constant $C_{4}$. 
Recalling the expression of $z$, we have, for each $a \geq 1$

$\left|\left\{(t, y) \in C_{j}^{+}(i): h^{\mu}(t, y)-M_{C_{j}(i)}^{\mu}(t-s)-\left(C_{2} r_{j}^{-2}+C_{3}\right)(t-s)-H_{C_{j}(i)}^{\mu}(0)>a\right\}\right| \leq \frac{C_{4}}{a}\left|C_{j}^{+}(i)\right|$.

Therefore, if we choose $a_{C_{j}(i)}=H_{C_{j}(i)}^{\mu}(0)$ and note the fact $r_{j} \leq 1 / 2$ again,

$$
\begin{aligned}
& \int_{C_{j}^{+}(i)} \sqrt{\left(h^{\mu}(t)-M_{C_{j}(i)}^{\mu}(t-s)-a_{C_{j}(i)}\right)^{+}} d y d t \\
\leq & \int_{0}^{4 r_{j}^{2}} \int_{B_{r_{j}}(x)} \sqrt{\left(h^{\mu}(t+s)-M_{C_{j}(i)}^{\mu}(t)-a_{C_{j}(i)}-\left(C_{2} r_{j}^{-2}+C_{3}\right) t\right)^{+}}+\sqrt{\left(C_{2} r_{j}^{-2}+C_{3}\right) t} d y d t \\
\leq & \int_{C_{j}^{+}(i), z \leq 1} \sqrt{z^{+}(t-s)} d y d t+\int_{C_{j}^{+}(i), z>1} \sqrt{z(t-s)} d y d t+2 \sqrt{C_{2}+C_{3}}\left|C_{j}^{+}(i)\right| \\
\leq & \int_{1}^{\infty} \frac{C_{4}\left|C_{j}^{+}(i)\right|}{a} d \sqrt{a}+\left(2+2 \sqrt{C_{2}+C_{3}}\right)\left|C_{j}^{+}(i)\right| \leq A\left|C_{j}^{+}(i)\right| .
\end{aligned}
$$

The other inequality can be proved by a completely symmetric procedure from (6.12) using $h(s-t, x)$ instead of $h(s+t, x)$ and this completes the proof for the lemma.

Our next step is to bound the random perturbation term $M^{\mu}$ in LEMMA 6.7

Lemma 6.8. Let $u$ be a non-negative super-solution to (1.1) in $[0,2] \times B_{1}$. For every $\epsilon>0$, there exist a constant $A$ depending only on $(n, l, \Lambda, \epsilon)$ and random variables $a_{j}(i)$ s such that for all $\mu>0$, inequalities (6.2) and (6.3) are satisfied for $f=h^{\mu}$ and any $C_{j}(i) \in \mathscr{C}^{\prime}$ on a set of at least probability $1-\epsilon$.

Proof. Since LemMA 6.7 gives two upper bounds almost surely, we only need to uniformly bound $M_{C_{j}(i)}^{\mu}(t)$ regardless of $\mu$.

We recall from REMARK 6.6 that there is a constant $J$ satisfying $\left\langle M_{C_{j}(i)}^{\mu}\right\rangle_{t} \leq J t$. Therefore for a large number $L>0$ and any $C_{j}(i) \in \mathscr{C}^{\prime}$, by [5, Lemma 3.1] we obtain,

$$
\mathbb{P}\left\{\sup _{0 \leq t \leq 4 r_{j}^{2}}\left|M_{C_{j}(i)}^{\mu}(t)\right| \geq L,\left\langle M_{C_{j}(i)}^{\mu}\right\rangle_{4 r_{j}^{2}} \leq 4 r_{j}^{2} J\right\} \leq \exp \left\{-L^{2} /\left(16 r_{j}^{2} J\right)\right\} .
$$

Since the second condition is always true, we have

$$
\mathbb{P}\left\{\sup _{0 \leq t \leq 4 r_{j}^{2}}\left|M_{C_{j}(i)}^{\mu}(t)\right| \geq L\right\} \leq \exp \left\{-L^{2} /\left(16 r_{j}^{2} J\right)\right\} .
$$

This inequality and LEMMA 6.7 tell us for some constant $\bar{A}$,

$$
\mathbb{P}\left\{\frac{1}{\left|C_{j}^{+}(i)\right|} \int_{C_{j}^{+}(i)} \sqrt{\left(h^{\mu}-a_{C_{j}(i)}\right)} d x d t>\bar{A}+\sqrt{L}\right\} \leq \exp \left\{-L^{2} /\left(16 r_{j}^{2} J\right)\right\},
$$

and a similar argument provides

$$
\mathbb{P}\left\{\frac{1}{\left|C_{j}^{-}(i)\right|} \int_{C_{j}^{-}(i)} \sqrt{\left(a_{C_{j}(i)}-h^{\mu}\right)+} d x d t>\bar{A}+\sqrt{L}\right\} \leq \exp \left\{-L^{2} /\left(16 r_{j}^{2} J\right)\right\} .
$$


In REMARK 6.3, we have denoted by $x_{j}$ the number of cubes with spatial radius $r_{j}$. Considering the event $\Theta(L)$ that there exists at least one of the $C_{j}(i)$ s such that (6.2) or (6.3) fails with $A+\sqrt{L}$, we have

$$
\mathbb{P}\{\Theta(L)\} \leq \sum_{j=0}^{\infty} 2 x_{j} \exp \left\{-L^{2} /\left(16 r_{j}^{2} J\right)\right\} \leq 2 \sum_{j=0}^{\infty} 4^{(n+3) j} \exp \left\{-16^{j} L^{2} /(4 J)\right\} .
$$

Therefore we can choose a sufficiently large $L$ to make $\mathbb{P}\{\Theta(L)\} \leq \epsilon$. This concludes our proof of the proposition with $A=\bar{A}+\sqrt{L}$.

Now we can finish the proof of PROPOSITION 6.1.

Proof of Proposition 6.1 For each $\epsilon>0$, LEMMA 6.8 provides us that on a set $\Omega^{\prime} \subseteq \Omega$ with probability at least $1-\epsilon,(6.2)$ and (6.3) hold for $h^{\mu}=-\log (u+\mu)$ with some random variables $a_{C_{j}(i)} \mathrm{S}$ and a constant $A$ depending on $\epsilon$ on all $C_{j}(i) \in \mathscr{C}^{\prime}$.

Applying Proposition 6.2 with $f=h^{\mu}$ on the set $\Omega^{\prime}$, we have for $v=\frac{b}{2 A}$,

$$
\mathscr{F}[u+\mu, v]_{D_{0}^{+}, D_{0}^{-}} \leq B^{2} v^{2}\left|D_{0}^{+}\right|\left|D_{0}^{-}\right|\left(\int_{0}^{\infty} e^{-\frac{b \alpha}{2 A}} d \alpha\right)^{2}
$$

on $\Omega^{\prime}$. The proposition is proved.

Remark 6.9. So far we have proved PROPOSITION 6.1 for $t=1 / 2$. For other values of $t$, we need to make the following changes.

- $C_{0}$ will be changed to $(0,2) \times B_{t}$.

- The relative positions of $C^{ \pm}, D^{ \pm}, I^{ \pm}$and $C$ in the division constructions of the cube collections will not change much and $C^{ \pm}$will still take the upper/lower halves of $C$. However, $D^{ \pm}$need to occupy the upper(lower) $t^{2} / 2$ portions and $I^{ \pm}$need to occupy the upper(lower) $t / 2$ portions.

- The division processes mentioned above also need to be finer. We need to choose an integer $\zeta$ sufficiently large and divide the cubes into $\zeta^{n+2}$ pieces instead of $4^{n+2}$. The criteria for the choice of $\zeta$ is to allow the proofs of LEMMA 6.4 and LEMMA 6.5 to go through.

- The smooth cut-off function in the third step needs to be 1 on $B_{t}$ and vanishes outside of $B_{(1+t) / 2}$.

The rest of the proof for the cases $t \neq 1 / 2$ is a verbatim repetition of the proofs in this and the next sections.

\section{ACKNOWLEDGEMENT}

The project was initially started by the author and Doctor Yu Wang (currently in Goldman Sachs) in 2014 upon the completion of [5]. Although the collaboration ended after the departure of Yu Wang, the discussion with him has helped clarify many confusions. His contribution to this project is greatly appreciated.

\section{REFERENCES}

[1] Carmona, R. and Rozovskii B. L. (ed.), Stochastic Partial Differential Equations: Six Perspectives, Mathematical Surveys and Monographs Series 64. Providence, RI: American Mathematical Society, 1998.

[2] Debussche, A., De Moor, S. and M. Hofmanova, A regularity result for quasilinear stochastic partial differential equations of parabolic type, arXiv:1401.6369

[3] Fabes, Eugene B., and Nicola Garofalo. Parabolic BMO and Harnack's inequality, Proceedings of the American Mathematical Society (1985): 63-69.

[4] Han, Qing, and Fanghua Lin. Elliptic partial differential equations. New York, 1997.

[5] Hsu, Elton P, Yu Wang, and Zhenan Wang. Stochastic De Giorgi iteration and regularity of stochastic partial differential equation, arXiv: 1312.3311, to appear in Annals of Probability.

[6] Krylov, N.V., An analytic approach to SPDEs, in Stochastic Partial Differential Equations: Six Perspectives, Mathematical Surveys and Monographs, vol. 64, pp. 185-242. AMS, Providence, RI 1999. 
[7] Moser, Jürgen, A Harnack inequality for parabolic differential equations, Communications on Pure and Applied Mathematics 17.1 (1964): 101-134.

[8] Mueller, Carl. On the support of solutions to the heat equation with noise, Stochastics and Stochastic Reports 37.4 (1991): 225-245.

[9] Pardoux, É., Equations aux Derivées Partielles Stochastiques Monotones, Thèse, Univ. Paris-Sud, 1975.

[10] Pardoux, É., Stochastic Partial Differential Equations, Lecture notes for the course given at Fudan University, 2007.

[11] Tessitore, G. and Zabczyk, J., Strict positivity for stochastic heat equations, Stochastic processes and their applications 77.1 (1998): 83-98. 